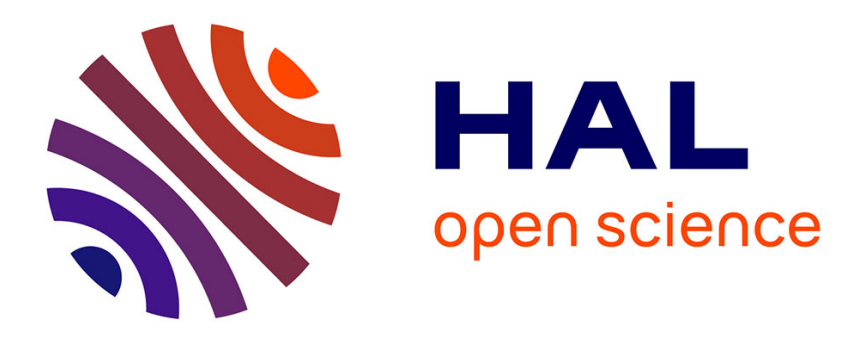

\title{
L'amplificateur CMOS dans les circuits à capacités commutées
}

F. Baillieu, R. Hermel

\section{To cite this version:}

F. Baillieu, R. Hermel. L'amplificateur CMOS dans les circuits à capacités commutées. Revue de Physique Appliquée, 1985, 20 (7), pp.465-482. 10.1051/rphysap:01985002007046500 . jpa-00245360

\section{HAL Id: jpa-00245360 https://hal.science/jpa-00245360}

Submitted on 1 Jan 1985

HAL is a multi-disciplinary open access archive for the deposit and dissemination of scientific research documents, whether they are published or not. The documents may come from teaching and research institutions in France or abroad, or from public or private research centers.
L'archive ouverte pluridisciplinaire HAL, est destinée au dépôt et à la diffusion de documents scientifiques de niveau recherche, publiés ou non, émanant des établissements d'enseignement et de recherche français ou étrangers, des laboratoires publics ou privés. 
Classification

Physics Abstracts

$12.20-12.90-25.70 \mathrm{C}$

\title{
L'amplificateur CMOS dans les circuits à capacités commutées (*)
}

\author{
F. Baillieu et R. Hermel \\ Groupe ESIEE, Chambre de Commerce et d'Industrie de Paris, 89, rue Falguière, 75015 Paris, France
}

(Reçu le 16 mai 1984, révisé le 15 novembre 1984, accepté le 25 mars 1985)

\begin{abstract}
Résumé. - La filière technologique CMOS représente sans doute la meilleure solution lorsqu'il s'agit de rassembler sur le même substrat des fonctions logiques et des fonctions analogiques moyenne fréquence utilisant le concept de la " commutation de capacités ".

L'amplificateur opérationnel est alors l'élément essentiel qui limite les performances de ces dernières (tensions de décalage, gain fini, taux de rejection, bande passante, vitesse de balayage, etc...). Fonctionnant sur charge capacitive, il est possible de retenir des amplificateurs se comportant en sortie, soit comme des sources de tension, soit comme des sources de courant.

La première solution est de maniement simple, mais ne conduit pas à des solutions optimums pour une technologie donnée.

La seconde solution permet d'obtenir des performances dynamiques meilleures, au prix toutefois d'une plus grande complexité de la conception qui ne peut être menée à bien que par l'emploi d'outils de simulation très performants.

L'objet de cet article est l'étude théorique comparative du comportement de ces deux classes d'amplificateurs dans des structures à capacités commutées et la description électrique de leur réalisation technologique.
\end{abstract}

\begin{abstract}
When one wants to integrate into single chip mid-frequency analog functions together with digital functions, the CMOS technology with switched capacitors appears to be the best solution.

The operational amplifier then becomes the main limiting factor for performance (offset, finite gain, rejection ratios, bandwidth, slew-rate, etc...).

However, two types of amplifiers may be used since the loads are just capacitors; i.e. amplifiers the output of which behave like voltage or current sources.

The voltage source option is simpler than the current source one, but implies lower performances. The latter option, however, is more difficult to implement.

The purpose of this paper is the theoretical study of the behaviour of these two types of amplifiers with switched capacitors as well as the electrical description of their technological realization.
\end{abstract}

\section{Généralités.}

Grâce à la fantastique croissance des densités d'intégration, il est désormais possible de rassembler sur une même " puce » de silicium, un ensemble complet de fonctions permettant ainsi l'émergence de véritables " systèmes intégrés sur silicium ".

Pour l'essentiel le traitement des signaux s'effectue de façon numérique car cette technique permet le développement d'algorithmes de calcul puissants, le stockage aisé de l'information et tolère bien les effets de la réduction des dimensions des composants.

Toutefois comme les grandeurs d'entrée-sortie de ces systèmes sont bien souvent de type analogique,

(*) Communication présentée aux Journées du G.C.I.S., Toulouse les 15 et 16 décembre 1983. il faut être capable d'intégrer sur le même substrat des fonctions analogiques d'interface performantes, car en définitive ce sont elles qui limiteront les possibilités réelles du système tout entier.

Par ailleurs, le traitement numérique, s'il est précis et performant, est aussi lourd à mettre en oeuvre. Il y a donc place pour des circuits de moyenne complexité, à objectifs certes plus modestes en matière de bruit, de précision... etc., mais opérant de manière purement analogique et réalisables en très grande quantité, à faible coût.

Plusieurs filières technologiques sont utilisables pour réaliser ces circuits où sont mêlées fonctions logiques et analogiques.

1.1 Filière bipolaire. - Les structures I2L permettent la réalisation des parties logiques tandis que les structures ECL permettent celles de dispositifs 
logiques très rapides et analogiques haute fréquence. Bien que la double optimisation soit délicate elle s'obtient néanmoins au prix d'une technologie assez pointue et d'une densité d'intégration moyenne.

1.2 FILIÈRE NMOS. - Très performante en logique, elle possède des possibilités réduites en linéaire par manque d'un transistor complémentaire [1].

1.3 Filière CMOS. - Le CMOS offre sans doute le meilleur compromis, car il permet la réalisation de structures logiques à faible consommation statique et à haut niveau d'intégration et qu'il est particulièrement bien adapté à la réalisation de fonctions analogiques moyenne fréquence en éliminant l'usage des résistances toujours délicates à intégrer et souvent encombrantes grâce à l'artifice de la commutation de capacités.

Par cette technique, il est possible de construire de façon élégante, des convertisseurs analogiques digitaux et digitaux analogiques, des filtres analogiques à échantillonnage, des références de tension, des amplificateurs d'instrumentation... etc.

L'élément clef des structures analogiques est toujours l'amplificateur; c'est la source première du bruit, des décalages, des limites des performances dynamiques. Aussi depuis quelques années, un grand nombre de schémas ont vu le jour dont il est possible d'effectuer une analyse comparative dans la perspective de leur utilisation dans les circuits utilisant la commutation de capacités [2-8].

1.4 Plan. - A partir de la description de l'intégrateur additionneur qui constitue la structure de base nous définirons les paramètres quasi statiques de l'amplificateur puis nous étudierons son comportement en commutation pour en fixer les paramètres dynamiques fondamentaux et extraire des éléments d'évaluation des structures amplificatrices sources de tension et amplificatrices sources de courant [1-8].

Nous serons alors en mesure de décrire de façon comparative les réalisations technologiques rencontrées dans la littérature.

\section{La structure de base.}

La structure de base de l'intégrateur additionneur pondéré est représentée (Fig. 1). Si l'amplificateur possède un gain infini et si la période de récurrence de l'horloge $(T)$ est faible par rapport à la période de la composante du signal à évolution la plus rapide, son comportement est décrit par l'expression :

$$
\begin{aligned}
V_{\text {out }}(t)=-\frac{C_{\mathrm{in}_{2}}}{C_{\text {out }}} V_{\mathrm{in}_{2}}(t) & -\frac{C_{\mathrm{in}_{3}}}{C_{\text {out }}} V_{\mathrm{in}_{3}}(t)- \\
& -\frac{1}{T} \int_{0}^{t} \frac{C_{\mathrm{in}_{1}}}{C_{\text {out }}} V_{\mathrm{in}_{1}}(\tau) \mathrm{d} \tau+K .
\end{aligned}
$$

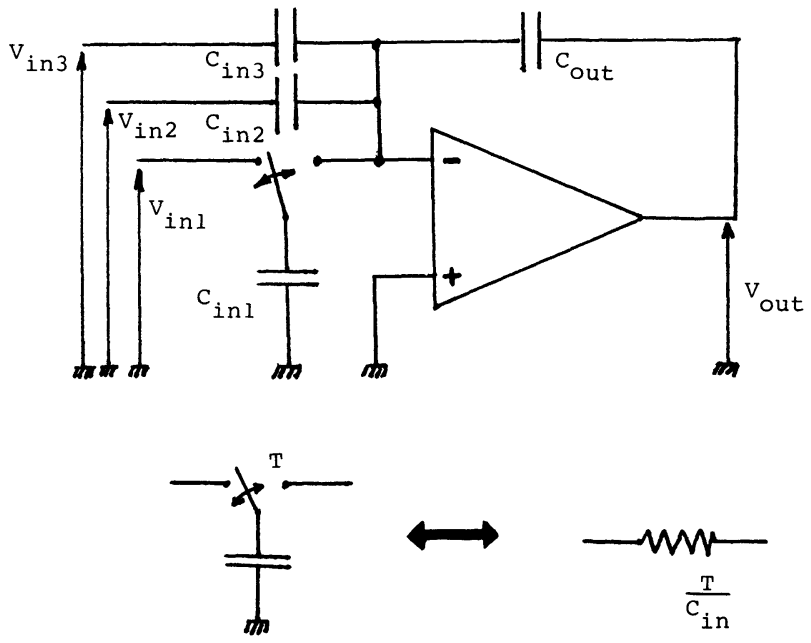

Fig. 1. - Montage de base dans les circuits à capacités commutées.

[Basic circuit.]

Il est à noter que la constante $K$ ne résulte pas seulement de la présence de l'intégrateur, elle apparaît aussi dans l'additionneur pondéré seul, contrairement à ce qui se passe dans son homologue à résistance; cela est dû au fait que l'impédance d'une capacité est infinie en continu. Cet aspect peut constituer un inconvénient dont il faut tenir compte au niveau de la synthèse du circuit.

En pratique cette structure est souvent modifiée à cause de la présence des capacités parasites (Fig. 2).

En effet, les capacités utiles $\left(C_{\mathrm{in}}, C_{\text {out }} ..\right)$ sont réalisées entre deux couches conductrices (Si-poly, Sipoly; Si-poly, métal ; métal, diffusion) selon la technologie, séparées par une couche isolante d'oxyde thermique. La capacité réelle est donc l'assemblage de la capacité utile et de deux capacités parasites. Celle constituée par l'électrode proche du substrat

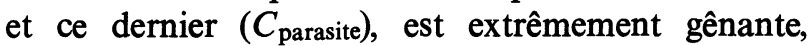
puisque comparable à la capacité utile. L'autre constituée par l'électrode la plus externe et l'environnement ( $\left.C_{\text {parasite }}^{\prime}\right)$ peut être bien réduite par une implantation soignée.

On élimine tous les effets parasites en substituant au schéma de principe de la figure 1 le schéma de la figure 3 .

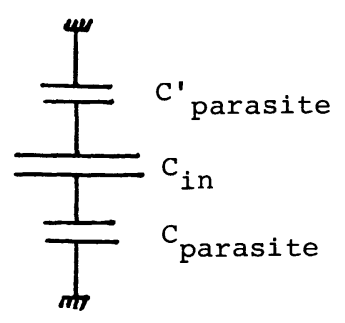

Fig. 2. 


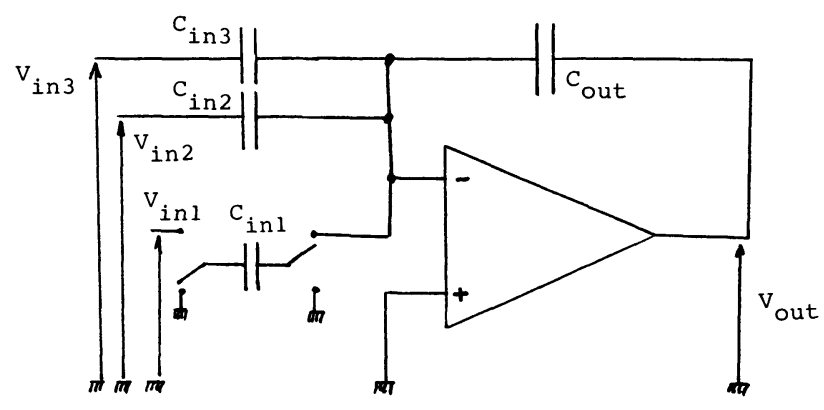

Fig. 3. - Montage de base éliminant l'effet des capacités parasites.

[Basic schematics avoiding parasitic effects.]

\section{Les caractéristiques quasi statiques de l'amplifica-} teur.

Un amplificateur opérationnel idéal est spécifié par les caractéristiques quasi statiques suivantes (Fig. 4) :

- Un gain différentiel quasi statique infini

$$
A_{0}=\infty \text {. }
$$

- Une impédance de sortie nulle

$$
R_{\text {out }}=0 \text {. }
$$

- Une impédance d'entrée infinie

$$
Z_{\mathrm{e}}=\infty \text {. }
$$

- Un taux de réjection du mode commun et des tensions d'alimentation infini.

Toutes ces contraintes ne doivent pas nécessairement être satisfaites par l'amplificateur utilisé dans les circuits à capacités commutées.

La résistance de sortie peut être élevée car l'amplificateur est chargé par une capacité quasiment sans fuite qui présente donc une impédance statique infinie. Ce choix conduit à une réelle simplification

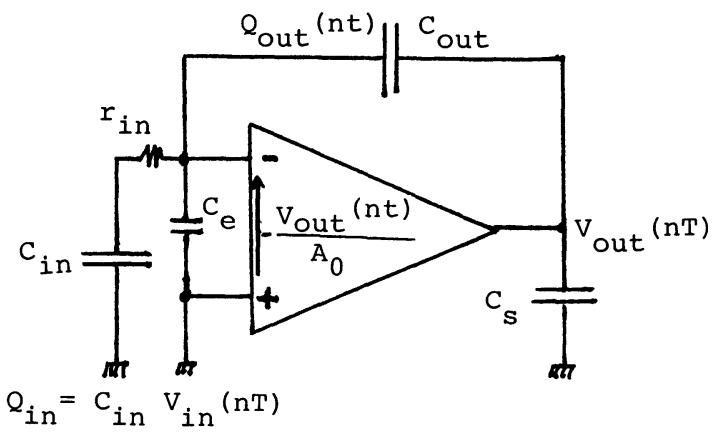

INITIAL

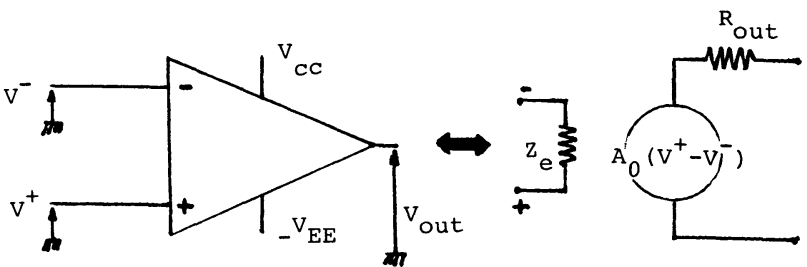

Fig. 4.

des montages en éliminant le buffer de sortie traditionnel des amplificateurs opérationnels et permet d'accroître la dynamique toute chose restant égale par ailleurs.

Le gain peut avoir une valeur finie $A_{0}$.

Le transfert de charge réel correspondant à une période d'échantillonnage est égal à (cf. Fig. 5) :

$$
\begin{array}{r}
\left(C_{\text {out }}+\frac{C_{\text {out }}+C_{\mathrm{e}}+C_{\text {in }}}{A_{0}}\right)\left(V_{\text {out }}(n+1) T-V_{\text {out }}(n T)\right)= \\
=-C_{\text {in }}\left(V_{\text {in }}(n T)-\frac{V_{\text {out }}(n T)}{A_{0}}\right)
\end{array}
$$

alors que le transfert idéal qui conduit à l'expression de définition est :

$C_{\text {out }}\left(V_{\text {out }}(n+1) T-V_{\text {out }}(n T)\right)=-C_{\text {in }} V_{\text {in }}(n T)(2 b i s)$

Le gain $A_{0}$ est choisi de valeur suffisamment élevée pour que son amplitude exacte n'ait pas d'importance significative, aussi :

$\frac{V_{\text {out }}(n T)}{A_{0} V_{\text {in }}(n T)} \ll 1 \rightarrow \frac{1}{A_{0} T} \int_{0}^{n T} \frac{\frac{C_{\text {in }}}{C_{\text {out }}} V_{\text {in }}(\tau) \mathrm{d} \tau}{V_{\text {in }} n T} \ll 1$.

En général, la qualité du transfert de charge n'est pas conditionnée par (2) et (3) mais par le comportement en impulsion de l'amplificateur.

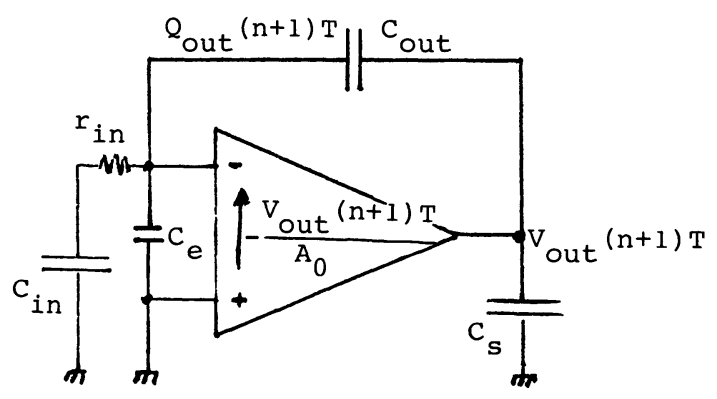

$$
Q_{\text {in final }}=\frac{-\left(C_{\text {in }}+C_{e}\right) V_{\text {out }}(n+1) T}{A_{0}}
$$

FINAL

Fig. 5. 
L'impédance d'entrée peut être capacitive et cette capacité peut être non stationnaire pour autant que la condition peu contraignante :

$$
C_{\mathrm{e}} \ll\left(A_{0}+1\right) C_{\text {out }}+C_{\text {in }}
$$

soit satisfaite.

La réjection du mode commun et des tensions d'alimentation devra être grande parce que le système est commandé par des horloges qui sont des sources de parasites de cette nature, mais là, nous commençons à aborder les aspects dynamiques de l'amplificateur.

En conclusion le schéma équivalent quasi statique de l'amplificateur est représenté par la figure 6 .

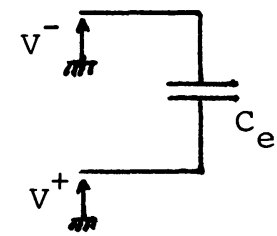

Fig. 6.

4. Les caractéristiques dynamiques de l'amplificateur. 4.1 GÉNÉRALITÉs. - Les contraintes les plus importantes résultent du fonctionnement en commutation, aussi seul va être étudié le comportement de l'intégrateur.

Le transfert de charge s'effectue en deux temps (Fig. 7).
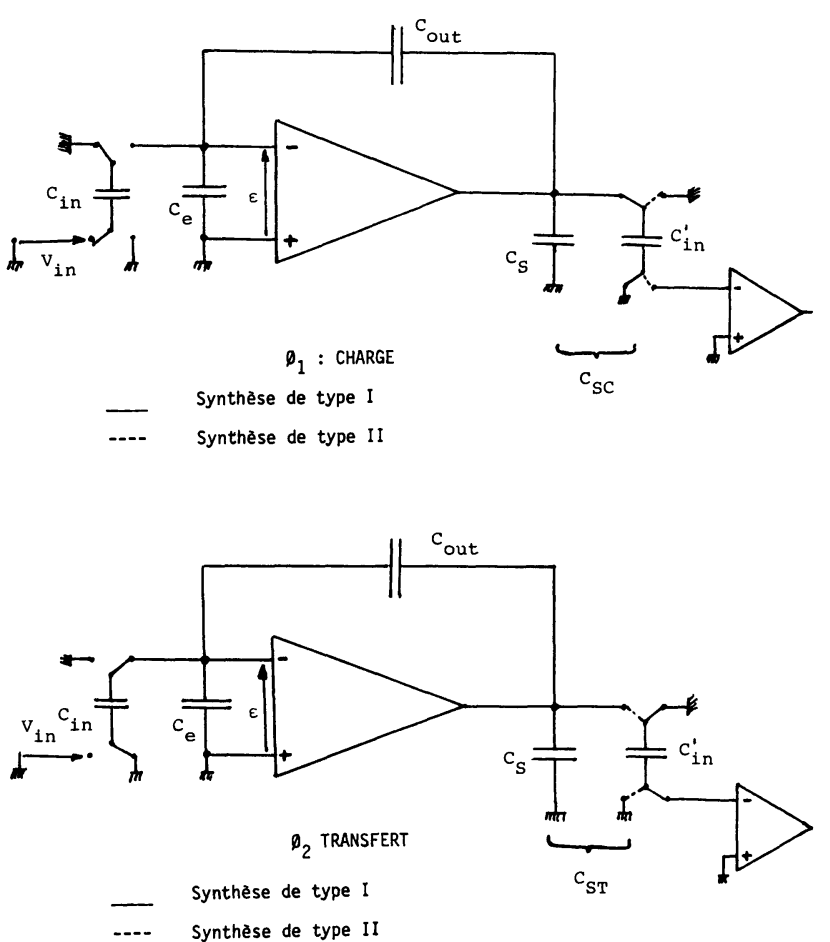

Fig. 7.
4.1.1 La première phase ou charge. - Elle correspond à la mise sous tension de $C_{\text {in }}$, le circuit est alors une mémoire analogique, la charge est constituée par la juxtaposition de trois à quatre capacités :

- la capacité $C_{\text {out }}$ de contre-réaction

- la capacité $C_{\mathrm{s}}$ qui est la capacité de sortie proprement dite de l'amplificateur

- la capacité d'entrée $C_{\mathrm{e}}$

- la capacité $C_{\mathrm{in}}^{\prime}$ dans certains types de synthèses.

4.1.2 La seconde phase ou transfert. - C'est durant ce laps de temps que la tension de sortie s'incrémente de la quantité $-\frac{C_{\text {in }}}{C_{\text {out }}} V_{\text {in }}$. Compte tenu de ce fait $C_{\text {in }}$ est en général très inférieur à $C_{\text {out }}$ pour que l'incrément par coup d'horloge soit réduit.

Chaque régime transitoire est lui-même constitué de deux périodes :

4.1.3 La vitesse de balayage (Fig. 8). - Durant la première période la tension $\varepsilon$ sort des limites de linéarité de l'amplificateur

$$
\begin{aligned}
& \varepsilon<-I^{+} / g \\
& \varepsilon>\quad I^{-} / g
\end{aligned}
$$

$I^{+}$et $I^{-}$sont respectivement les limites positives et négatives d'un certain courant. Ce sont les extrema du courant de sortie des amplificateurs " source de courant " et d'un courant intermédiaire dans les amplificateurs " source de tension ".

C'est la phase de balayage caractérisée par une vitesse de balayage ou " slew rate "

$$
\left|\frac{\mathrm{d} V_{\text {out }}}{\mathrm{d} t}\right|=\left|\frac{I^{+} \text {ou } I^{-}}{C^{*}}\right| \text {. }
$$

La valeur de $C^{*}$ est liée au choix de l'amplificateur. C'est la capacité interne de compensation dans les amplificateurs "source de tension " et la capacité de charge dans les amplificateurs "source de courant" comme nous allons le montrer dans l'étude approfondie du balayage.

4.1.4 Le temps d'établissement (Fig. 8). - La tension $\varepsilon$ est alors comprise entre les limites de linéarité

$$
-\frac{I^{+}}{g .}<\varepsilon<\frac{I^{-}}{g} .
$$

L'amplificateur possède une fonction de transfert souvent assez voisine dans les deux phases de fonctionnement le " transfert ", la " charge ".

En très grand signal c'est la vitesse de balayage qui limite les performances des circuits alors qu'en petit et moyen signal c'est plutôt le temps d'établissement. Compte tenu, en général, de la faible valeur de $C_{\text {in }}$ par rapport à $C_{\text {out }}$ ce temps doit être particulièrement optimisé. 

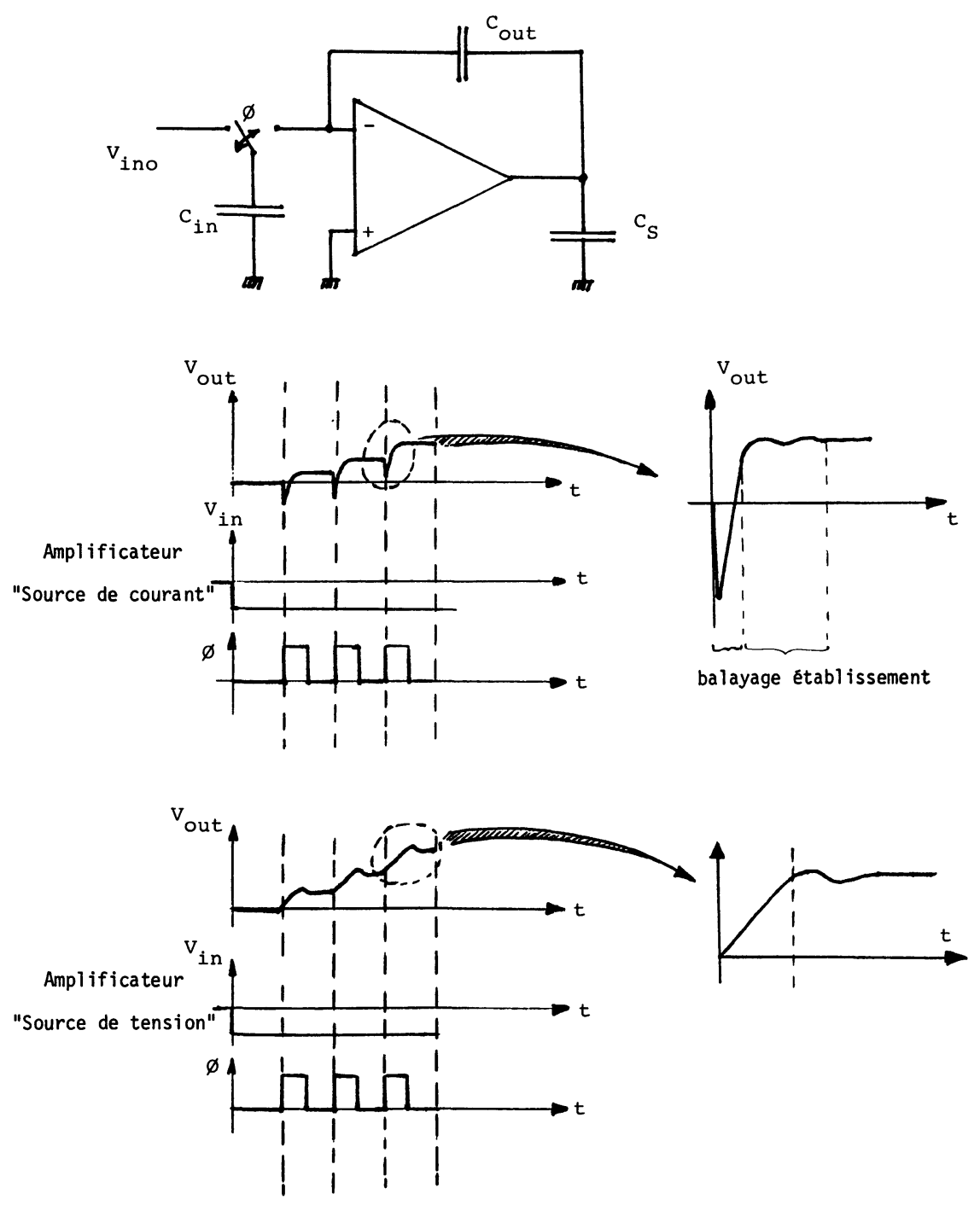

Fig. 8.

\section{Etude approfondie du balayage.}

La phase de balayage est caractérisée par la vitesse de balayage

$$
\frac{\mathrm{d} V_{\text {out }}}{\mathrm{d} t}=\longrightarrow \begin{aligned}
& I^{+} / C^{*} \\
& -I^{-} / C^{*}
\end{aligned}
$$

selon le signe de $V_{\text {in }}$. La capacité $C^{*}$ dépend du type d'amplificateur retenu.

5.1 L'AMPLIFICATEUR SOURCE DE TENSION. - Cet amplificateur dont le prototype est donné figure 9 et le schéma équivalent différentiel figure 10 se comporte dans une large bande de fréquence comme un amplificateur source de tension.

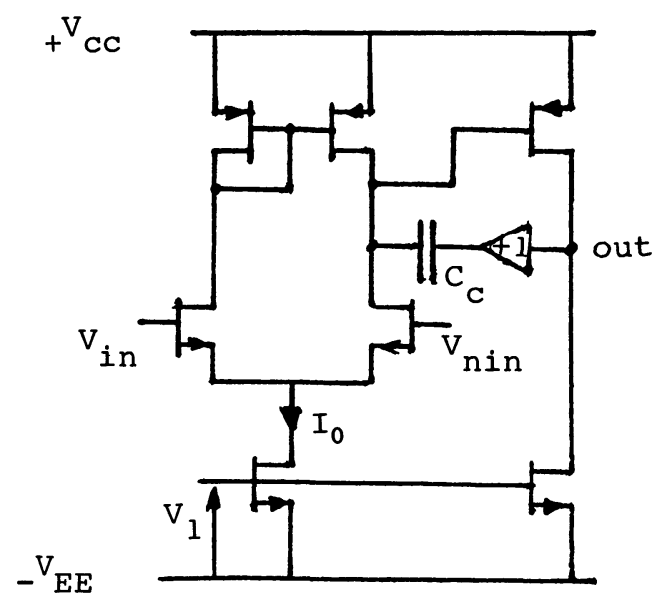

Fig. 9. - Le prototype de l'amplificateur source de tension. [Voltage source amplifier prototype.] 


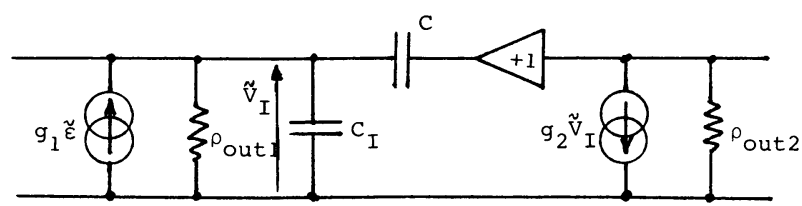

Fig. 10.

$C$ représente la capacité de contre-réaction interne qui assure la stabilité du système en boucle fermée. En régime de balayage le premier étage est saturé, il faut substituer à la source de courant $g_{1} \varepsilon$ une source de courant constant $I^{+}$ou $I^{-}$suivant le signe de $\varepsilon$. Le schéma équivalent est représenté (Fig. 11).

En négligeant le courant dérivé par la capacité de faible valeur $C_{\mathrm{I}}$ nous obtenons après trois transformations le schéma pratique (Figs. 12a, b, c) où il apparaît clairement que l'amplificateur se comporte comme une source de tension de Miller de faible résistance interne $1 / g_{2}$.

Le temps de balayage durant la phase de transfert peut s'estimer à l'aide des figures $13 \mathrm{a}, \mathrm{b}, \mathrm{c}$, où $r_{\text {in }}$ représente les interrupteurs. En négligeant les constantes de temps :

$$
\tau_{\text {in }}=r_{\text {in }} C_{\text {in }} \text { et } \quad \tau_{\mathrm{s}}=C_{\mathrm{s}} / g_{2}
$$

il vient

$$
\frac{\mathrm{d} V_{\text {out }}}{\mathrm{d} t}=+\frac{I^{+} \text {ou } I^{-}}{C}
$$

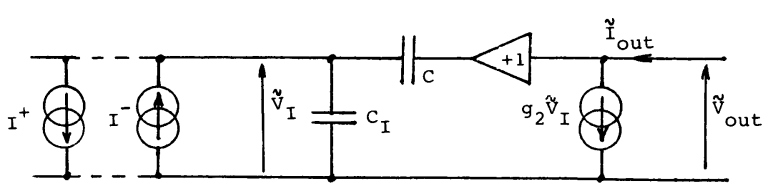

Fig. 11.

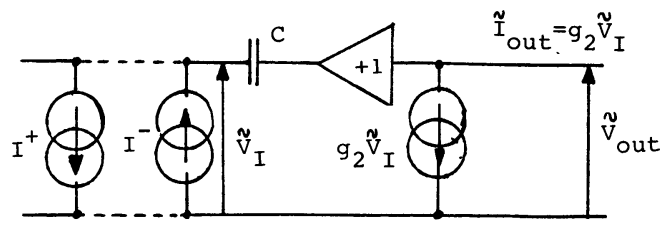

Fig. 12a.

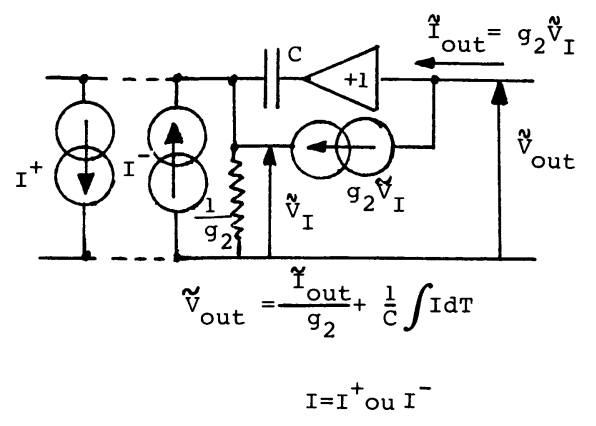

Fig. 12b.

Fig. 12c.

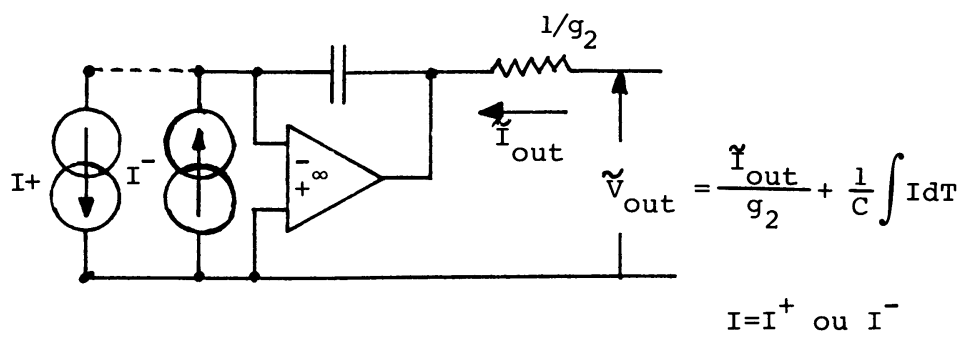

donc

$$
\begin{aligned}
\tau_{\text {transf }} & =\frac{C}{C_{\text {out }}} \cdot\left|\frac{C_{\text {in }} \cdot V_{\text {in }}(n T)-\left(C_{\text {in }}+C_{\mathrm{e}}+C_{\text {out }}\right) \varepsilon_{\text {final }}}{I^{+} \text {ou } I^{-}}\right| \\
& =C \cdot\left|\frac{\left\{V_{\text {out }}(n+1) T-V_{\text {out }}(n T)\right\}+\frac{C_{\text {in }}+C_{\mathrm{e}}+C_{\text {out }}}{C_{\text {out }}} \varepsilon_{\text {final }}}{I^{+} \text {ou } I^{-}}\right| .
\end{aligned}
$$

Pour des évaluations d'ordre de grandeur il est possible de négliger le terme en $\varepsilon_{\text {final }}$ dans l'expression (8). Ceci revient à supposer la transconductance $g_{2}$ infinie.
5.2 L'AMPlificateur SOURCE DE COURANT. - Les prototypes de ces structures sont l'amplificateur à transconductance (O.T.A.), l'amplificateur cascode complémentaire et leurs variantes, tous décrits ultérieurement. 


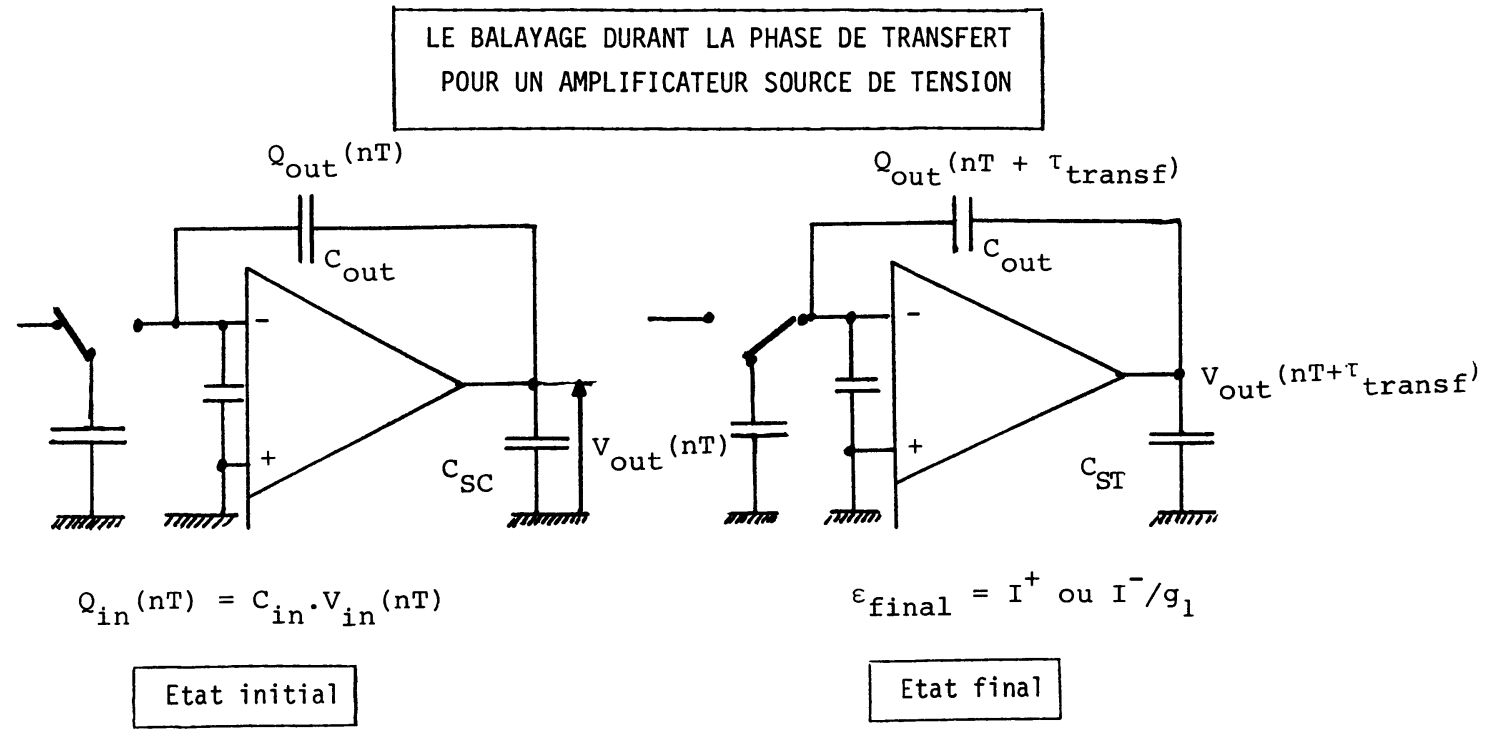

Fig. 13a.
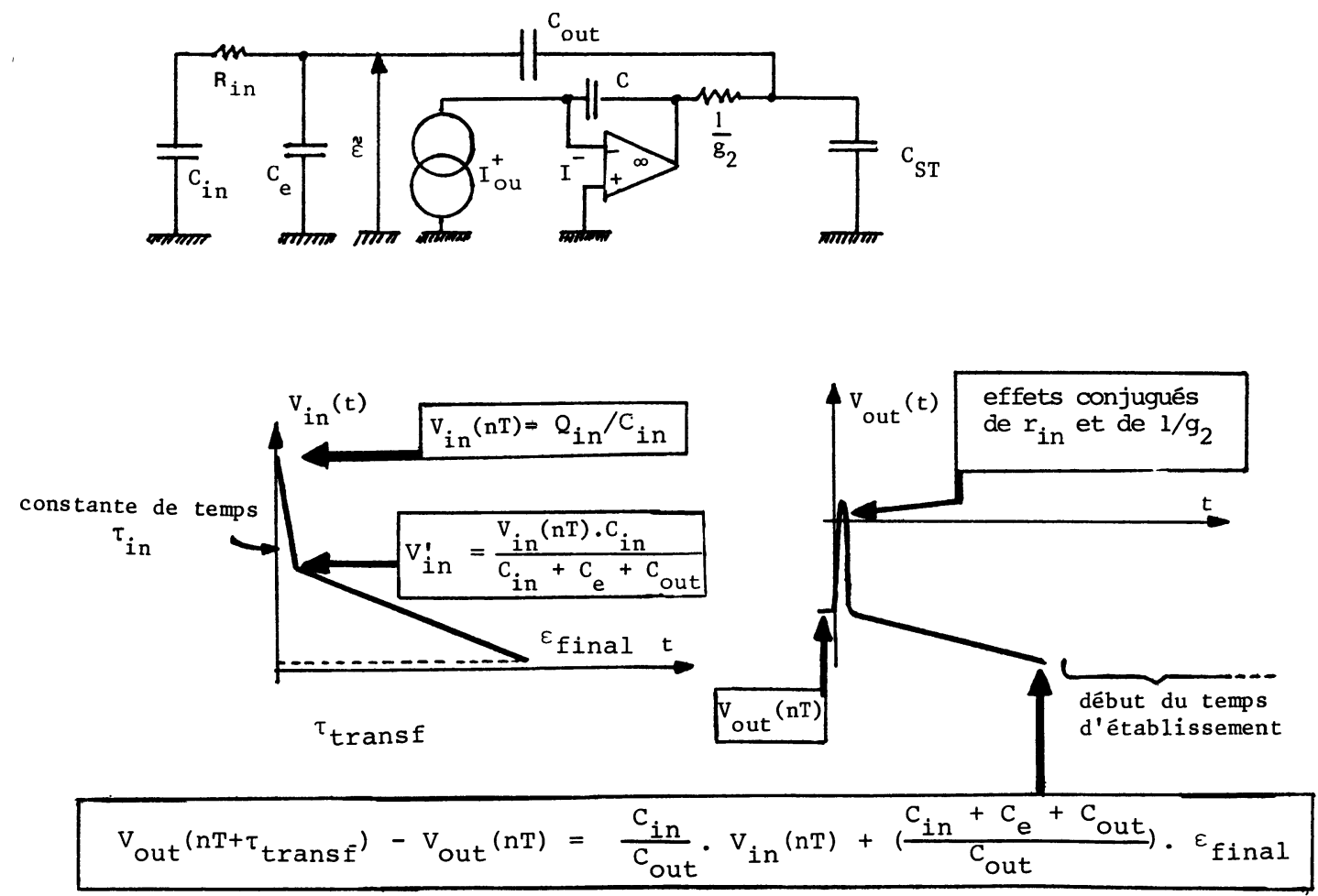

Fig. $13 b$.

Fig. 13c.

Compte tenu de l'effet de saturation en régime de balayage, le schéma dynamique se réduit à la figure 14 . Le temps de balayage durant la phase de transfert peut s'estimer à l'aide des figures $15 \mathrm{a}, \mathrm{b}$ et $16 \mathrm{a}, \mathrm{b}$ avec :

$$
\left(\frac{\mathrm{d} V_{\text {out }}}{\mathrm{d} t}\right)_{I}=\left(I^{+} \text {ou }-I^{-}\right) /\left(C_{\mathrm{ST}}+\frac{C_{\text {out }} \cdot\left(C_{\text {in }}+C_{\mathrm{e}}\right)}{\left.C_{\text {out }}+C_{\text {in }}+C_{\mathrm{e}}\right)}\right) .
$$

Pour calculer simplement cette durée, en négligeant la constante de temps $\tau_{\text {in }}$, il convient de remarquer que la quantité de charge contenue par la portion du circuit en trait fort des figures $15 \mathrm{a}$ et $\mathrm{b}$ ne varie que grâce à 

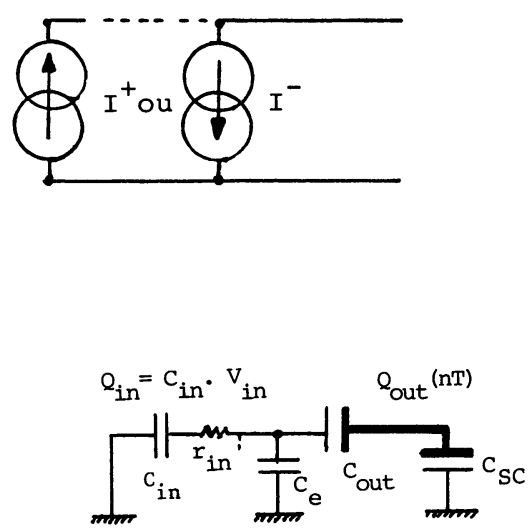

$\mathrm{v}_{\text {out }}(\mathrm{nT}) \cdot \mathrm{C}_{\mathrm{SC}}=\mathrm{Q}_{\mathrm{SC}}$

Etat initial $\longleftarrow$ Fig. 14

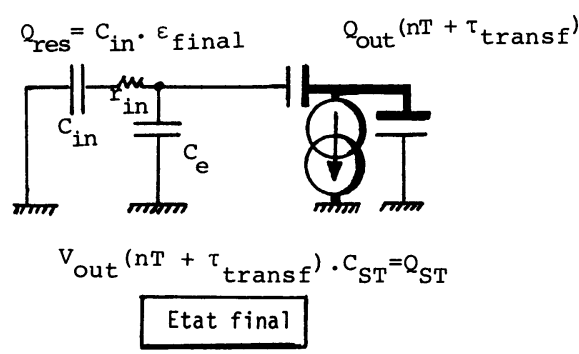

Fig. $15 b$.

Fig. 15a.

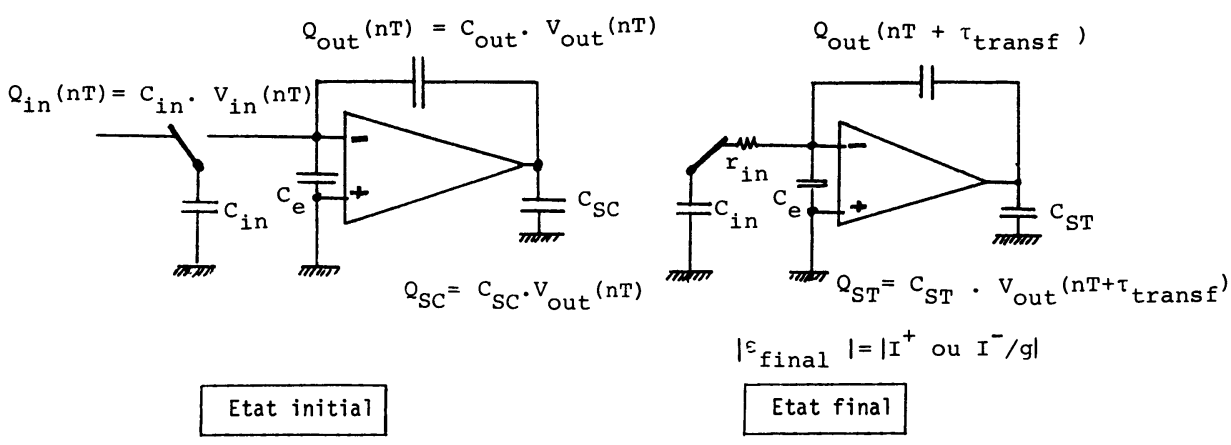

Fig. 16a.

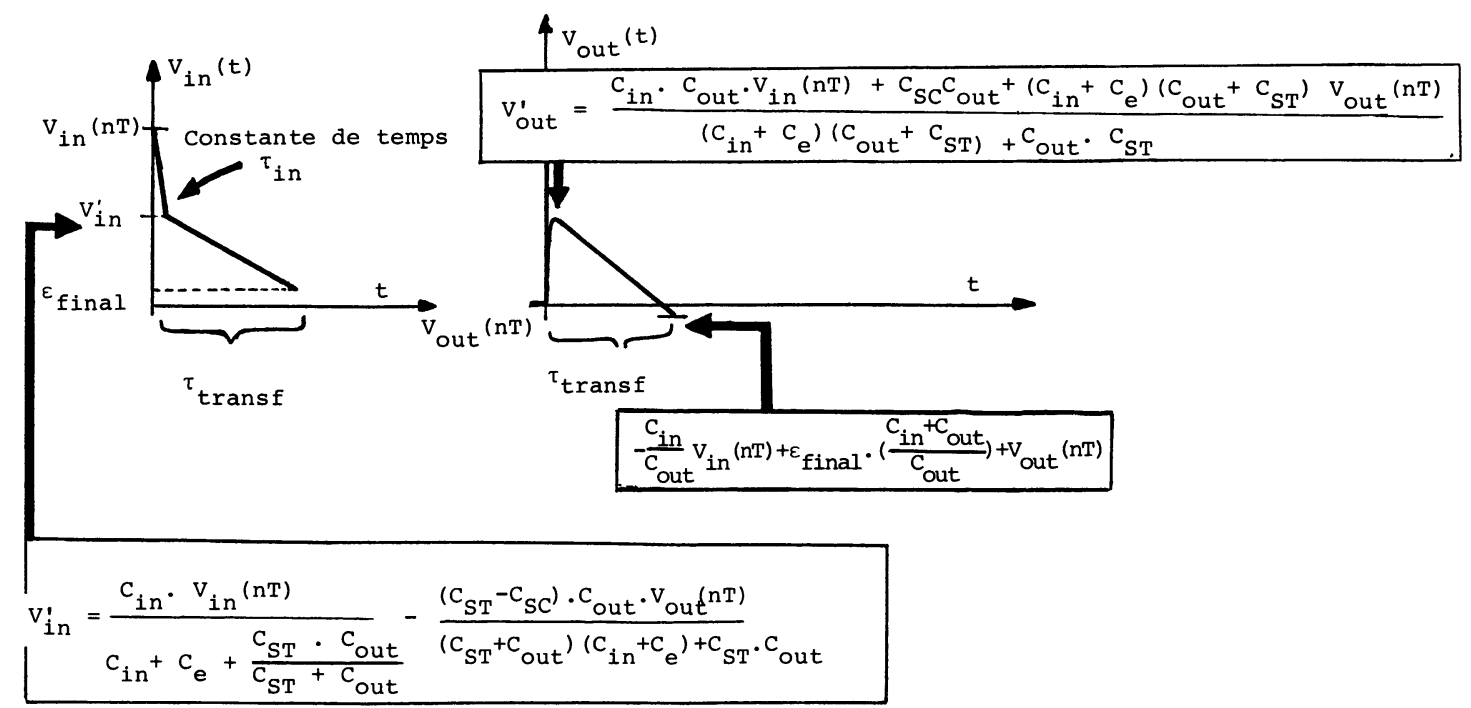

Fig. 16b. 
l'apport de la source de courant $I^{+}, I^{-}$, or :

$$
\int_{0}^{\tau_{\text {transf }}} I \mathrm{~d} t=Q_{\text {out }}\left(n T+\tau_{\text {transf }}\right)-Q_{\text {out }}(n T)+Q_{\mathrm{ST}}-Q_{\mathrm{SC}}
$$

aussi :

$$
\begin{aligned}
& \tau_{\text {transf }}=\frac{\left(\frac{C_{\text {out }}+C_{\mathrm{ST}}}{C_{\text {out }}}\right) \cdot C_{\text {in }} V_{\text {in }}(n T)-\left(C_{\text {in }}+C_{\mathrm{e}}+\frac{C_{\text {out }} C_{\mathrm{ST}}}{C_{\text {out }}+C_{\mathrm{ST}}}\right) \cdot \varepsilon_{\text {final }}}{I^{+} \text {ou } I^{-}}- \\
& -\frac{\left(C_{\mathrm{ST}}-C_{\mathrm{SC}}\right) \cdot\left(\frac{C_{\text {out }}}{C_{\text {out }}+C_{\mathrm{ST}}}\right) \cdot V_{\text {out }}(n T)}{I^{+} \text {ou } I^{-}} \\
& \tau_{\text {transf }}=\frac{\left(C_{\text {out }}+C_{\mathrm{ST}}\right) \cdot\left(V_{\text {out }}(n+1) T-V_{\text {out }}(n T)\right)+\left(\frac{C_{\mathrm{ST}}-C_{\mathrm{SC}}}{C_{\text {out }}+C_{\mathrm{ST}}}\right) V_{\text {out }}(n T)}{I^{+} \text {ou } I^{-}}+ \\
& +\frac{\left(\frac{C_{\text {in }}+C_{\mathrm{e}}}{C_{\text {out }}}+\frac{C_{\mathrm{ST}}}{C_{\text {out }}+C_{\mathrm{ST}}}\right) \cdot \varepsilon_{\mathrm{final}}}{I^{+} \text {ou } I^{-}} .
\end{aligned}
$$
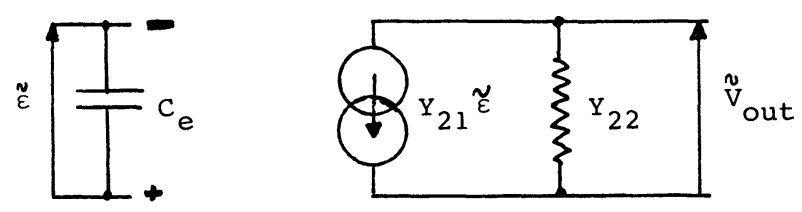

Fig. 17.
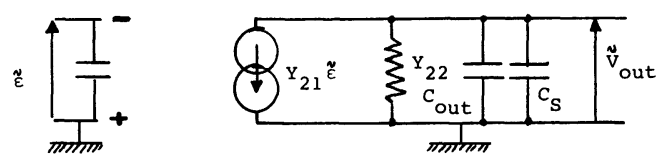

Gain en tension

Fig. $18 \mathrm{a}$

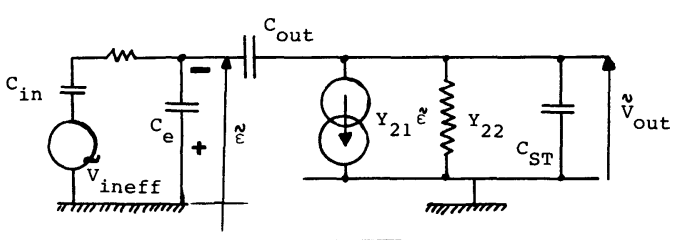

Fig. $18 \mathrm{~b}$.

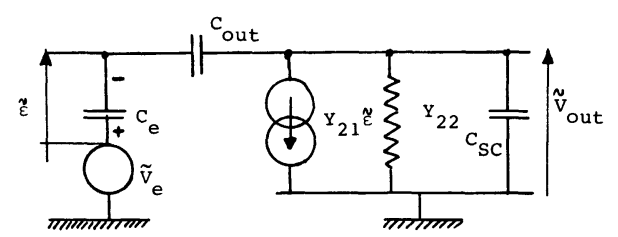

Charge

Fig. 18c.

ReVUE de PHYSIQUE APPLIQUÉE. - T. 20, No 7, JULLLET 1985

\section{Gain en tension}

$$
\frac{V_{\text {out }}(p)}{\varepsilon(p)}=-\frac{Y_{21}}{Y_{22}+p\left(C_{\text {out }}+C_{S}\right)}=\frac{Y_{21}}{Y_{22}+Y_{\text {out }}}
$$

Fonction de transfert : phase de transfert

$$
\frac{V_{\text {out }}(p)}{V_{\text {in eff }}(p)}=-\frac{C_{\text {in }}}{C_{\text {out }}} \cdot \frac{A^{\prime}(p)}{A^{\prime}(p)+\frac{C_{\text {out }}+C_{\mathrm{e}}+C_{\text {in }}}{C_{\text {out }}}}
$$

avec

$$
A^{\prime}(p)=\frac{Y_{21}-p C_{\text {out }}}{Y_{22}+p\left(C_{\text {out }}+C_{\mathrm{ST}}\right)} .
$$

Fonction de transfert : phase de charge

$$
\frac{V_{\text {out }}(p)}{V_{\mathrm{e}}(p)}=-\frac{C_{\text {out }}}{C_{\mathrm{e}}} \cdot \frac{A^{\prime \prime}(p)}{A^{\prime \prime}(p)+\frac{C_{\text {out }}+C_{\mathrm{e}}}{C_{\text {out }}}}
$$

avec

$$
A^{\prime \prime}(p)=\frac{Y_{21}-p C_{\mathrm{out}}}{Y_{22}+p\left(C_{\mathrm{out}}+C_{\mathrm{SC}}\right)}
$$

Il est à noter qu'il n'y a pas de régime transitoire correspondant à la période de charge si deux inté- 
grateurs consécutifs sont horlogés en opposition de phase. Par ailleurs les critères de stabilité sont voisins dans les deux modes de fonctionnement, transfert et charge. Aussi allons-nous limiter notre étude à celle de l'établissement durant la phase de transfert.

Il existe en fait deux sortes d'amplificateurs, les premiers où dans un vaste domaine de fréquence :

$$
\left|Y_{22}\right|<\left|p\left(C_{\text {out }}+C_{\mathrm{ST}}\right)\right|
$$

se comportent plutôt comme des sources de courant. Leur fonction de transfert s'écrit alors pour la phase de transfert :

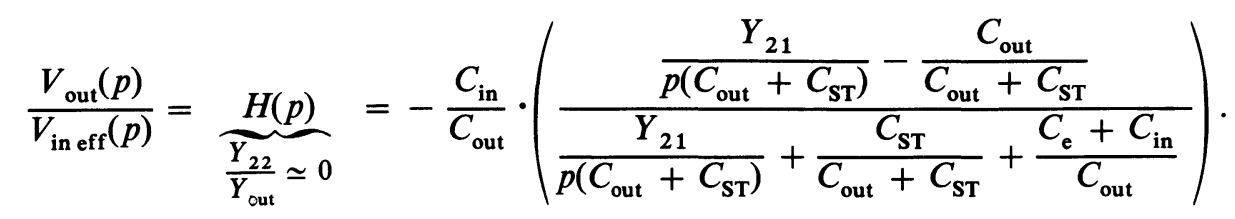

Les seconds où dans un vaste domaine de fréquence

$$
\left|p\left(C_{\text {out }}+C_{\mathrm{ST}}\right)\right|<\left|Y_{22}\right|
$$

se comportent plutôt comme des sources de tension.

Leur fonction de transfert s'écrit alors durant la phase de transfert :

$$
\frac{V_{\text {out }}(p)}{V_{\text {in eff }}(p)}=\frac{H(p)}{\frac{Y_{\text {out }}}{Y_{22}} \simeq 0}=-\frac{C_{\text {in }}}{C_{\text {out }}} \cdot\left(\frac{\frac{Y_{21}}{Y_{22}}}{\frac{Y_{21}}{Y_{22}}+\frac{C_{\text {out }}+C_{\mathrm{e}}+C_{\text {in }}}{C_{\text {out }}}}\right) \text {. }
$$

Les comportements en transitoire de ces deux fonctions de transfert sont extrêmement différents comme le met en évidence l'étude qualitative suivante.

D'une manière générale le gain en tension des amplificateurs peut se mettre sous la forme :

$$
\frac{V_{\mathrm{out}}(p)}{\varepsilon(p)}=-A(p)=-\frac{Y_{21}}{Y_{22}+p\left(C_{\mathrm{out}}+C_{\mathrm{ST}}\right)}=-\frac{A_{0}}{\left(1+\frac{p}{\omega_{\mathrm{p}_{1}}}\right)\left(1+\frac{p}{\omega_{\mathrm{p} 2}}\right)} .
$$

Ce qui correspond au diagramme de Bode représenté

(Fig. 19).

$\omega_{\mathbf{p}_{1}}$ est le pôle basse fréquence $\left(f_{\mathbf{p}_{1}}=\omega_{\mathbf{p}_{1}} / 2 \pi\right.$ varie de quelques $\mathrm{Hz}$ à quelques centaines de $\mathrm{Hz}$ ). $\omega_{\mathrm{p}_{2}}$ est le pôle haute fréquence $\left(f_{\mathrm{p}_{2}}=\omega_{\mathrm{p}_{2}} / 2 \pi\right.$ varie de quelques $\mathrm{MHz}$ à quelques centaines de $\mathrm{MHz}$ dans les technologies les plus performantes).

En négligeant le fait que le gain en tension statique est fini, ce qui n'a pas d'effet sur la stabilité, $A(p)$ se simplifie pour devenir :

$$
A(p) \simeq \frac{A_{0} \omega_{\mathrm{p}_{1}}}{p\left(1+P / \omega_{\mathrm{p}_{2}}\right)}=\frac{\omega_{\mathrm{T}}}{P\left(1+P / \omega_{\mathrm{p}_{2}}\right)}
$$

$\omega_{\mathbf{p}_{2}}$ est supérieur à $\omega_{\mathrm{T}}$, aussi pour une analyse qualitative comparative des deux types de fonctions de transfert (12bis) et (13bis) il est possible de pousser plus loin encore l'approximation en posant :

$$
A(p) \simeq \frac{\omega_{\mathrm{T}}}{P}
$$

Attaquons alors par un même créneau les deux fonctions de transfert $H(p)$ (expressions (13) et
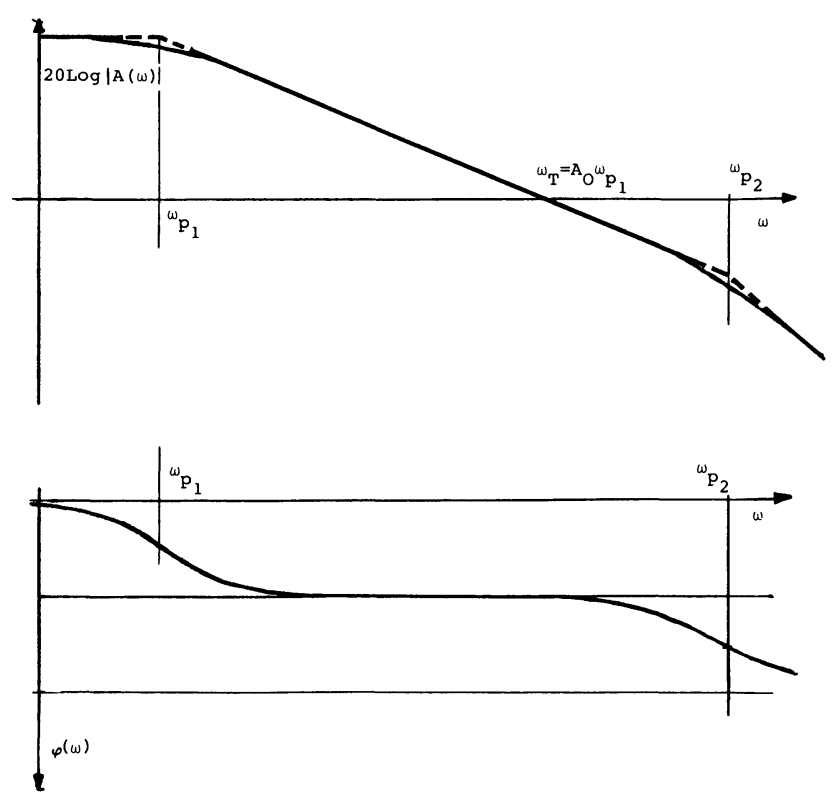

Fig. 19. 
(13bis)) à $\omega_{\mathrm{T}}$ constant, dans les deux cas particuliers fondamentaux définis ci-dessous :

$1 \mathrm{er}$ cas :

$$
C_{\text {in }}<C_{\text {out }} ; C_{\mathrm{ST}}<C_{\text {out }} ; C_{\mathrm{e}} \ll C_{\text {out }} .
$$

Amplificateur source de courant

$$
\begin{aligned}
V_{\text {out }}(t) & =-\frac{V_{\text {in eff }} C_{\text {in }}}{C_{\text {out }}}\left(1-\left(1+\frac{1}{\omega_{\mathrm{T}} \tau}\right) \cdot \exp \left\{-\frac{t}{\tau}\right\}\right. \\
\tau & =\frac{1}{\omega_{\mathrm{T}}} \cdot \frac{C_{\text {in }}+C_{\mathrm{ST}}}{C_{\text {out }}+C_{\mathrm{ST}}} .
\end{aligned}
$$

Amplificateur source de tension

$$
V_{\text {out }}(t)=-\frac{V_{\text {in eff }} C_{\text {in }}}{C_{\text {out }}}\left(1-\exp \left\{-\omega_{\mathrm{T}} t\right\}\right) .
$$

$2^{\mathrm{e}}$ cas :

$$
C_{\text {in }}>C_{\text {out }} ; C_{\mathrm{ST}}<C_{\text {out }} ; C_{\mathrm{e}} \ll C_{\text {in }} .
$$

Dans ces conditions :

$$
\begin{aligned}
& \begin{array}{l}
V_{\text {out }}(t)= \\
\text { Amp. tension }
\end{array} \\
& \quad V_{\text {ineff }} \frac{C_{\text {in }}}{C_{\text {out }}}\left(1-\exp \left(-\omega_{\mathrm{T}} \frac{C_{\text {out }}}{C_{\text {out }}+C_{\text {in }}}\right)^{t}\right) \\
& \text { Amp. courant }
\end{aligned}
$$

Le premier cas, qui caractérise le mode de fonctionnement le plus fréquent de l'intégrateur à capacités commutées, met en évidence les différences de comportement des deux types d'amplificateurs.

L'amplificateur source de courant doit toujours être chargé entre sortie et masse par une capacité de faible valeur $\left(C_{\mathrm{s}} \mathrm{min}\right)$ pour rester stable même lorsque $C_{\text {in }}$ est déconnecté (phase de charge). En effet la constante de temps $\tau$ doit toujours être supérieure à $1 / \omega_{\mathrm{p} 2}$.

L'utilisation de l'amplificateur de courant dans l'intégrateur engendre un parasite de commutation d'amplitude d'autant plus élevée qu'il est de durée brève lors de chaque transfert de charge (Fig. 20).

Ces deux aspects sont en faveur de l'amplificateur source de tension. Cet avantage est toutefois largement contrebalancé par le fait que le temps de commutation total (balayage plus établissement) de l'intégrateur à amplificateur source de courant, peut être rendu totalement indépendant de $C_{\text {out }}$. Ceci permet de banaliser ce type d'amplificateur et va dans le sens de la conception automatisée de circuits analogiques à partir de cellules standards précaractérisées.

Cette propriété remarquable est bien mise en évidence dans l'étude qui suit.

\section{Réalisations technologiques CMOS.}

6.1 AMPLIFICATEURS SOURCE DE TENSION ET SOURCE DE COURANT. - Dans ce paragraphe nous vérifions sur les schémas équivalents des structures prototypes des amplificateurs opérationnels que ceux-ci appartiennent à deux classes : les amplificateurs source de tension et les amplificateurs source de courant.

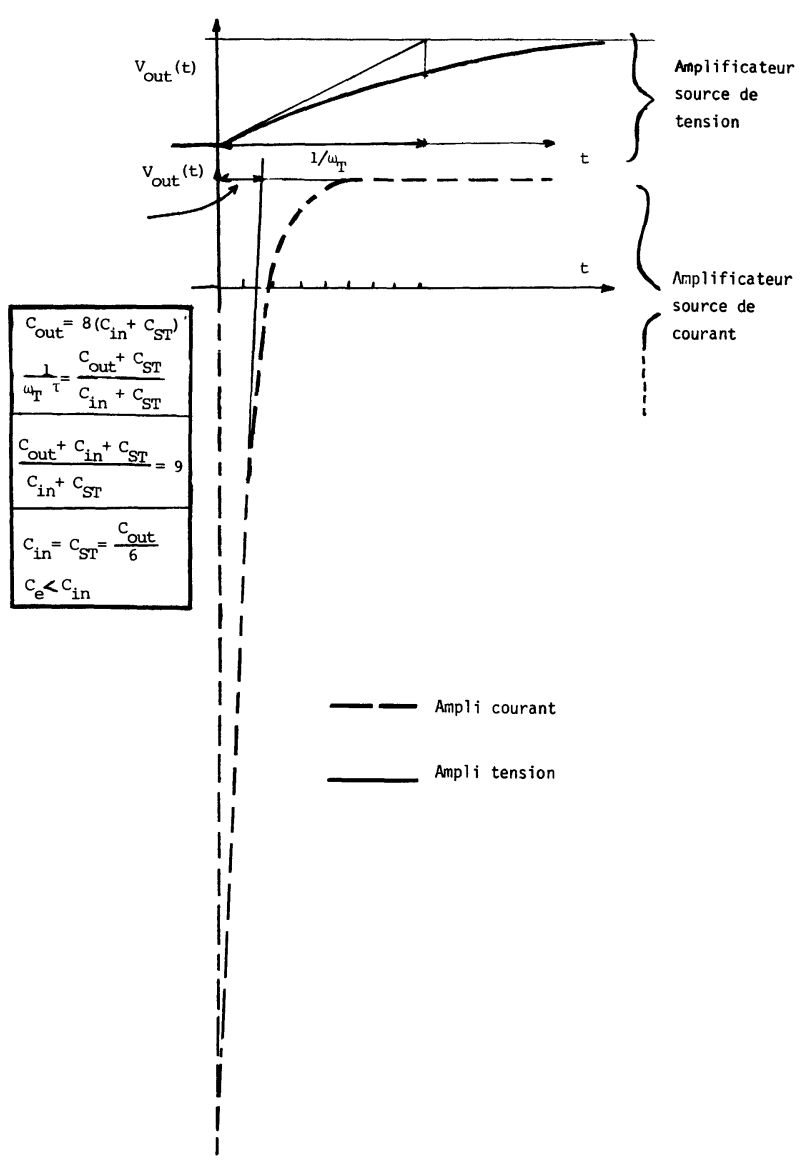

Fig. 20. - Comparaison des régimes transitoires des amplificateurs source de tension et source de courant en régime d'établissement.

[Transient behaviour of voltage source and current source amplifiers.] 


\subsubsection{Source de tension (Fig. 21).}

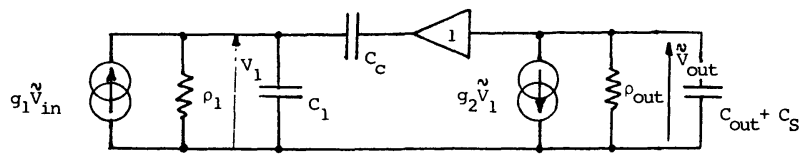

Fig. 21.

Le gain en tension $A_{\mathrm{V}}=\frac{V_{\text {out }}}{V_{\text {in }}}$ est donné par :

$$
A_{\mathrm{v}}=\frac{-Y_{21}}{Y_{22}+Y_{\text {out }}}
$$

avec $\quad Y_{21}=\frac{g_{1} g_{2} \rho_{1}}{1+\rho_{1}\left(C_{\mathrm{c}}+C_{1}\right) P}$

$$
\begin{gathered}
Y_{22}=\frac{1}{\rho_{\text {out }}}+\frac{P C_{\mathrm{c}} g_{2} \rho_{1}}{1+\rho_{1}\left(C_{\mathrm{c}}+C_{1}\right) P}=g_{2} \frac{C_{\mathrm{c}}}{C_{\mathrm{c}}+C_{1}} \\
Y_{\text {out }}=P\left(C_{\text {out }}+C_{\mathrm{s}}\right) .
\end{gathered}
$$

L'amplificateur se comporte comme une source de tension si $\left|Y_{22}\right| \gg\left|Y_{\text {out }}\right|$.

D'où

$$
\left|\frac{1}{\rho_{\text {out }}}+\frac{P C_{\mathrm{c}} g_{2} \rho_{1}}{1+\rho_{1}\left(C_{\mathrm{c}}+C_{1}\right) P}\right| \gg\left|P\left(C_{\text {out }}+C_{\mathrm{s}}\right)\right| \text {. }
$$

Cette condition est vérifiée pour une fréquence comprise entre

$$
f_{1}=\frac{1}{2 \pi \rho_{1}\left(\bar{C}_{1}+C_{\mathrm{c}}\right)} \text { et } f_{2}=\frac{g_{2}}{2 \pi\left(C_{\mathrm{out}}+C_{\mathrm{S}}\right)} .
$$

La courbe de Bode de la fonction de transfert a l'allure dessinée sur la figure 2 où $f_{\mathrm{p}_{1}}$ et $f_{\mathrm{p}_{2}}$ sont les pôles de la fonction de transfert

et

$$
f_{1}=g_{2} \rho_{\text {out }} f_{\mathrm{p}_{1}}
$$

$$
f_{2}=f_{\mathrm{p}_{2}} \text {. }
$$

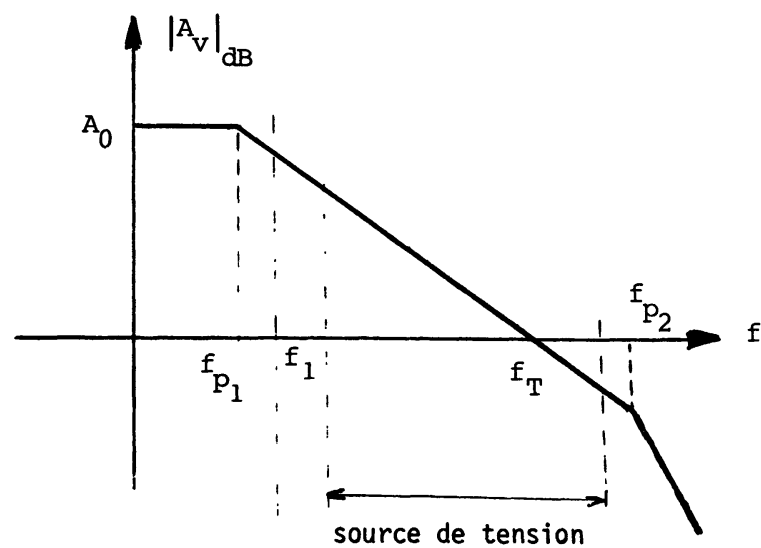

Fig. 22.
6.1.2 Source de courant (Fig. 23).
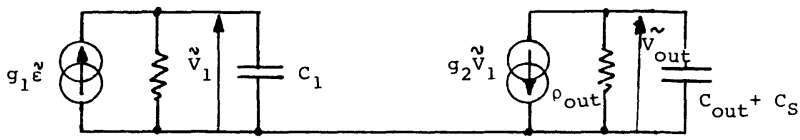

Fig. 23.

$$
\begin{gathered}
Y_{21}=\frac{g_{1} g_{2} \rho_{1}}{1+p C_{1} \rho_{1}} \quad Y_{22}=\frac{1}{\rho_{\text {out }}} \\
Y_{\text {out }}=P\left(C_{\text {out }}+C_{\mathrm{S}}\right) .
\end{gathered}
$$

La condition pour que l'amplificateur de la figure 23 se comporte comme une source de courant est $\left|Y_{22}\right| \ll\left|Y_{\text {out }}\right|$.

D'où :

$$
\left|\frac{1}{\rho_{\text {out }}}\right| \ll\left|P\left(C_{\text {out }}+C_{\mathrm{S}}\right)\right| .
$$

Cette condition est satisfaite pour une fréquence supérieure à

$$
f_{0}=\frac{1}{2 \pi \rho_{\text {out }}\left(C_{\text {out }}+C_{\mathrm{s}}\right)}
$$

$f_{0}$ correspond au pôle basse fréquence $f_{\mathrm{p}_{1}}$ pour ce type d'amplificateur (Fig. 24).

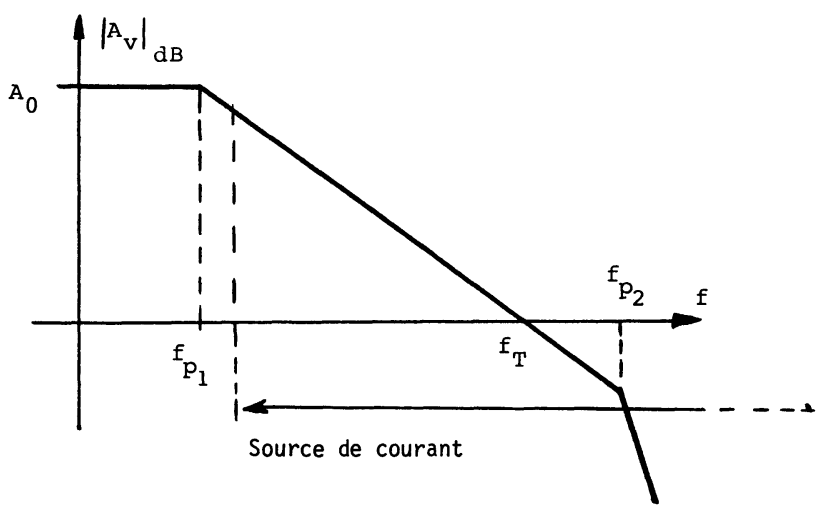

Fig. 24.

\subsection{AmplifiCATEUR DE TYPE SOURCE DE TENSION.}

6.2.1 Amplificateur " traditionnel 》 (Fig. 25). - Le suiveur placé en série avec la capacité de compensation est nécessaire pour assurer la stabilité de l'amplificateur jusqu'à un gain en boucle fermée unité. 


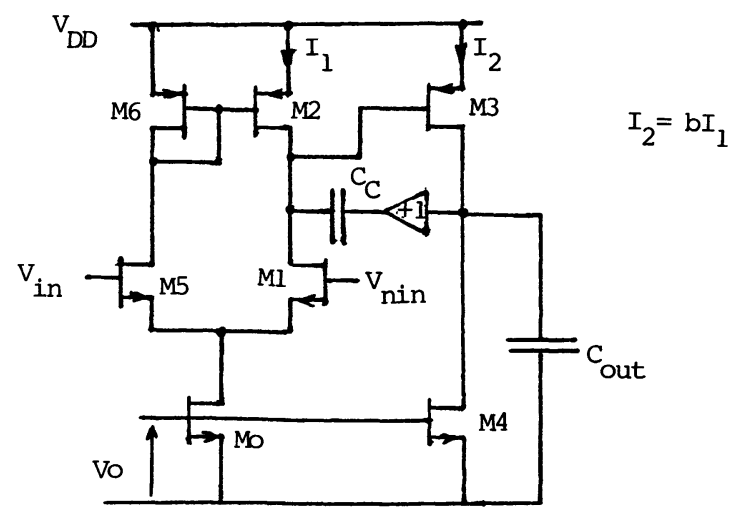

Fig. 25.

La fonction de transfert en mode différentiel est de la forme :

$$
A_{\mathrm{v}}=A_{0} \frac{\left(1-\frac{P}{\omega_{\mathrm{z}_{1}}}\right)\left(1-\frac{P}{\omega_{\mathrm{z}_{2}}}\right)\left(1-\frac{P}{\omega_{\mathrm{z}_{3}}}\right)}{\left(1+\frac{P}{\omega_{\mathrm{p}_{1}}}\right)\left(1+\frac{P}{\omega_{\mathrm{p}_{2}}}\right)\left(1+\frac{P}{\omega_{\mathrm{p}_{3}}}\right)}
$$

avec

$$
\begin{aligned}
A_{0} & =g_{m_{1}} g_{m_{3}} \rho_{2} \rho_{\mathrm{out}} & \omega_{\mathrm{p}_{1}} & =\frac{1}{g_{m_{3}} C_{\mathrm{c}} \rho_{2} \rho_{\mathrm{out}}} \\
\omega_{\mathrm{p} 2} & =\frac{g_{m_{3}}}{C_{\mathrm{out}}} & \omega_{\mathrm{p}_{3}} & =\frac{g_{m_{2}}}{C_{1}} \\
\omega_{\mathrm{z}_{i}} & =\frac{g_{m_{i}}}{C_{\mathrm{GD} i}} i=1,2,3 & \omega_{\mathrm{T}} & =A_{0} \omega_{\mathrm{p}_{1}}=\frac{g_{m_{1}}}{C_{\mathrm{c}}}
\end{aligned}
$$

où

$$
\begin{aligned}
& C_{1}=C_{\mathrm{GD} 5}+C_{\mathrm{DB} 5}+C_{\mathrm{DB} 6}+C_{\mathrm{GS} 6}+C_{\mathrm{GS} 2}+C_{\mathrm{GD} 2} \\
& \begin{array}{l}
\tau_{\text {établissement }}=\frac{1}{\omega_{\mathrm{T}}}=\frac{C_{\mathrm{c}}}{9 m_{1}} \propto C_{\text {out }} \\
\tau_{\text {balayage }} \\
\qquad 1 / C_{\text {out }}
\end{array}
\end{aligned}
$$

Les notations relatives aux paramètres des transistors sont détaillées en annexe.

L'expression des éléments du schéma équivalent en fonction des paramètres technologiques donne :

$$
\begin{aligned}
A_{0} & \propto \frac{1}{\sqrt{b} I_{0_{1}}} & \omega_{\mathrm{T}} & =\frac{\sqrt{2 \beta_{1} I_{0_{1}}}}{C_{\mathrm{c}}} \\
\omega_{\mathrm{p}_{2}} & \propto \frac{\sqrt{b I_{0_{1}}}}{C_{\mathrm{out}}} & S R & =\frac{I_{0_{1}}}{C_{\mathrm{c}}}
\end{aligned}
$$

$S R$ désigne la vitesse de balayage $\left(\frac{\mathrm{d} V_{\text {out }}}{\mathrm{d} t}\right)$ (slew-rate).
La condition de stabilité $\omega_{\mathrm{T}}<\omega_{\mathrm{p}_{2}}$ (marge de phase $\geqslant 45^{\circ}$ ) montre que la valeur de la capacité de compensation $C_{\mathrm{c}}$ doit être choisie en fonction de la valeur de la charge $C_{\text {out }}$.

D'autre part, nous avons vu plus haut que l'écart entre $\omega_{T}$ et $\omega_{p_{2}}$ détermine la forme de la réponse transitoire de l'amplificateur. Cette double condition peut donc être un inconvénient car l'optimisation de la conception du circuit conduit à une plage de valeurs réduite pour la capacité de charge, à capacité de compensation fixée.

6.2.2 Amplificateur traditionnel à étage cascode (Fig. 26). - Les transistors $\mathbf{M}_{2}$ et $\mathbf{M}_{7}$ permettent de connecter directement la capacité de compensation $C_{\mathrm{c}}$ sans suiveur.

La fonction de transfert de cet amplificateur présente

- un pôle basse fréquence $\omega_{\mathrm{p}_{1}}=\frac{1}{C_{\mathrm{c}} g_{m_{4}} \rho_{3} \rho_{\text {out }}}$

- deux pôles très voisins l'un de l'autre

$$
\omega_{\mathrm{p} 2} \text { et } \omega_{\mathrm{p}^{\prime}} \simeq \sqrt{\frac{\left(g_{m_{2}}+g_{m_{2}}^{\prime}\right) g_{m_{4}}}{C_{2}^{*}\left(C_{\mathrm{out}}+C_{\mathrm{c}}\right)}} .
$$

La proximité de ces deux pôles entraîne une rotation de phase très rapide, donc un risque d'instabilité plus important que dans le circuit précédent.

Néanmoins, cette structure permet, en dimensionnant convenablement les transistors $\mathbf{M}_{2}$ et $\mathbf{M}_{4}$, d'obtenir une fréquence de transition plus élevée que dans le montage classique (rapport 5 à 10 environ). Ceci entraîne une valeur de la capacité de compensation plus faible, donc une surface moindre. Ce montage permet d'utiliser dans les circuits à capacités commutées une fréquence d'horloge plus grande que dans celui décrit paragraphe 2.1, mais sa conception en vue d'optimiser la réponse transitoire est plus délicate.

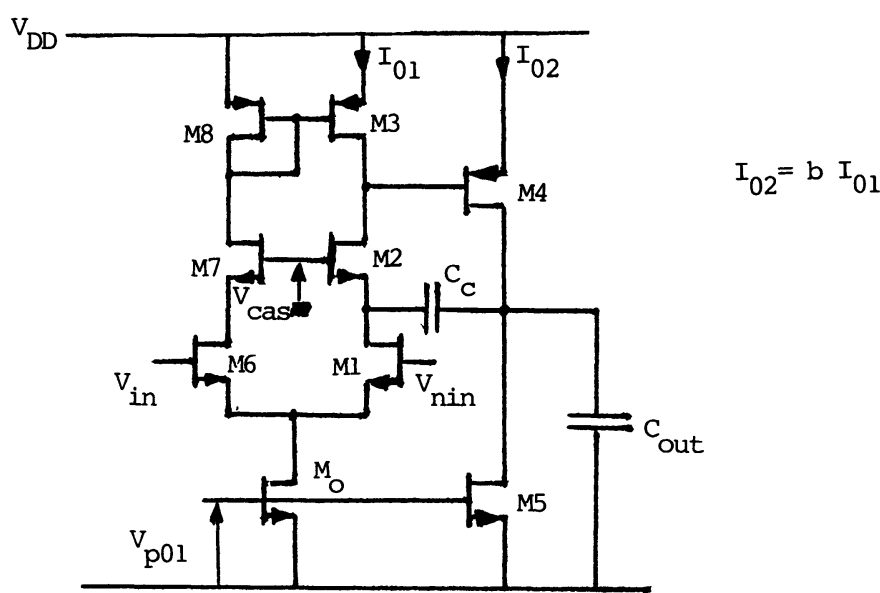

Fig. 26. 
Les valeurs des paramètres importants de cet amplificateur sont les suivantes :

$$
\begin{aligned}
& A_{0}=g_{m_{1}} g_{m_{4}} \rho_{3} \rho_{\text {out }} ; \quad A_{0} \propto \frac{1}{\sqrt{b} I_{0_{1}}} \\
& S R=\frac{I_{0_{1}}}{C_{\mathrm{c}}} \frac{I_{0_{2}}}{C_{\text {out }}}=\frac{b I_{0_{1}}}{C_{\text {out }}} \\
& \omega_{\mathrm{T}}=\frac{g_{m_{1}}}{C_{\mathrm{c}}}=\frac{\sqrt{2 \beta_{1} I_{0_{1}}}}{C_{\mathrm{c}}} \\
& \omega_{\mathrm{p}_{2}} \simeq \omega_{\mathrm{p}_{2}}=\sqrt{\frac{\left(g_{m_{1}}+g_{m_{2}}\right) g_{m_{4}}}{C_{2}^{*}\left(C_{\mathrm{c}}+C_{\mathrm{out}}\right)}} \\
& \omega_{\mathrm{p}_{2}} \propto \sqrt{\frac{b I_{0_{1}}}{C_{2}^{*}\left(C_{\mathrm{c}}+C_{\text {out }}\right)}}
\end{aligned}
$$

$C_{2}^{*}$ est la capacité parasite vue depuis la grille de $\mathrm{M}_{4}$.

$$
\begin{aligned}
& \tau_{\text {établissement }}=\frac{1}{\omega_{\mathrm{T}}}=\frac{C_{\mathrm{c}}}{9 m_{1}} \\
& \tau_{\text {balayage }} \propto C_{\mathrm{c}}+C_{\text {out }} \\
& \propto 1 / C_{\text {out }}
\end{aligned} \mid C_{\text {out }}>C_{\text {in }} .
$$

6.3 AMPLifiCATEURS DE TYPE SOURCE DE COURANT 6.3.1 Amplificateur à transconductance (O.T.A.) (Fig. 27). - Ce montage est basé sur le principe de la recopie de courants grâce aux sources miroirs de courant $\mathbf{M}_{2}, \mathbf{M}_{3}$ et $\mathbf{M}_{6}, \mathbf{M}_{7}$. Aussi dans un large domaine de fréquence, le courant disponible dans la charge, $C_{\text {out }}$ est proportionnel à la tension de commande $\left(V_{\text {nin }}-V_{\text {in }}\right)$. D'où l'appellation d'amplificateur à transconductance. La faible impédance vue des nœuds 2 et $2^{\prime}$ rend illusoire l'usage de l'effet Miller pour assurer la compensation en fréquence. Cette dernière est donc assurée par la capacité de charge elle-même.

Valeurs importantes :

où

$$
\begin{aligned}
\omega_{\mathrm{p} 1} & =\frac{1}{\rho_{\text {out }} C_{\text {out }}} \text { avec } \rho_{\text {out }}=\rho_{3} \| \rho_{4} \\
A_{0} & =\frac{g_{m_{1}} g_{m_{3}} \rho_{\text {out }}}{g_{m_{2}}} \\
\omega_{\mathrm{T}} & =\frac{g_{m_{1}} g_{m_{3}}}{g_{m_{2}} C_{\text {out }}}=\frac{b \sqrt{2 \beta_{1} I_{0_{1}}}}{C_{\text {out }}} \\
S R & =\frac{b I_{0_{1}}}{C_{\text {out }}} \quad \omega_{\mathrm{p}_{2}}=\frac{g_{m_{2}}}{C_{1}}=\frac{\sqrt{2 \beta_{2} I_{0_{1}}}}{C_{1}}
\end{aligned}
$$

$$
\begin{gathered}
C_{1}=C_{\mathrm{GD} 1}+C_{\mathrm{DB} 1}+C_{\mathrm{DB} 2}+C_{\mathrm{GS} 3} \\
\tau_{\text {établissement }}=\frac{C_{\mathrm{in}}+C_{\mathrm{ST}}}{b g_{m_{1}}}=\text { Cte } . \\
\tau_{\text {balayage }} \quad C_{\text {indépendant de } C_{\text {out }}}>C_{\text {in }}
\end{gathered}
$$

Cet amplificateur est stable dès lors que la capacité de charge est suffisamment grande devant la capacité interne $C_{1}$, le même circuit peut donc être utilisé avec un grand nombre de charges capacitives différentes, ce qui se révèle particulièrement intéressant

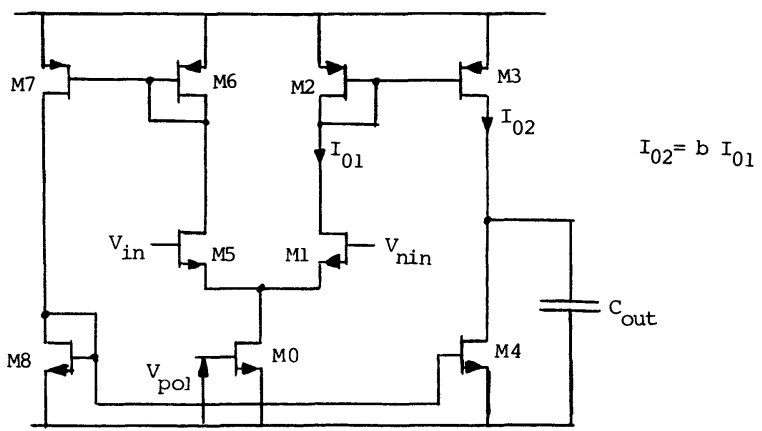

Fig. 27.

dans le cadre d'une conception automatique de systèmes complets.

L'amplificateur opérationnel peut alors être considéré comme une cellule précaractérisée.

6.3.2 Amplificateur cascode complémentaire (Fig. 28).

\section{Valeurs importantes :}

$$
\begin{gathered}
A_{0}=g_{m_{1}} \rho_{4} \quad A_{0} \propto \frac{\sqrt{I_{0}}}{I_{0}^{\prime}-I_{0}} \\
\omega_{\mathrm{T}}=\frac{g_{m_{1}}}{C_{\text {out }}}=\frac{\sqrt{2 \beta_{1} I_{0_{1}}}}{C_{\text {out }}} \\
\tau_{\text {établissement }}=\frac{C_{\text {in }}+C_{\mathrm{ST}}}{g_{m_{1}}}=\mathrm{Cte} \\
\left.\tau_{\text {balayage }}\right|_{\text {indépendant de } C_{\text {out }}} C_{\text {out }}>C_{\text {in }} \\
\omega_{\mathrm{p}_{2}}=\frac{g_{m_{3}}+g_{m_{3}}^{\prime}}{C_{1}} \quad \omega_{\mathrm{p}_{2}} \propto \frac{\sqrt{I_{0}^{\prime}-I_{0}}}{C_{1}}
\end{gathered}
$$

avec

$$
\begin{gathered}
C_{1}=C_{\mathrm{GD} 1}+C_{\mathrm{DB} 1}+C_{\mathrm{DB} 2}+C_{\mathrm{GD} 2}+C_{\mathrm{GS} 3}+C_{\mathrm{SB} 3} \\
S R=\frac{I_{0}^{\prime}-I_{0}}{C_{\mathrm{out}}} .
\end{gathered}
$$

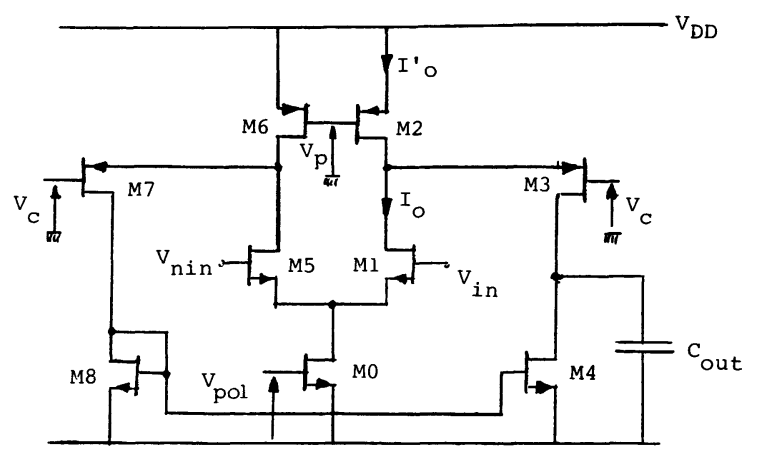

Fig. 28 . 
Comme l'O.T.A., cet amplificateur est naturellement stable sur charge capacitive. De plus, il est plus facile de régler les différentes valeurs des fréquences de coupure grâce à la possibilité de contrôler indépendamment les sources de courant $I_{0}$ et $I_{0}^{\prime}$.

Cet amplificateur apparaît donc plus souple à utiliser que l'O.T.A.

Les amplificateurs source de courant ont un gain statique plus faible que les amplificateurs source de tension. Ceci n'est pas un réel inconvénient car la caractéristique la plus importante pour l'utilisation en capacités commutées est la position relative de $\omega_{\mathrm{T}}$ et $\omega_{\mathrm{p} 2}$. Le gain statique doit néanmoins être suffisamment élevé pour que l'erreur statique sur un intégrateur soit négligeable. Il est possible de l'augmenter en utilisant un étage de sortie de type cascode. La figure 33 représente l'application de cette amélioration aux amplificateurs à transconductance.

\subsubsection{Amélioration des structures existantes.}

6.3.3.1 Structures différentielles (Fig. 30). - Toutes les structures décrites plus haut sont des structures dissymétriques. Il en résulte une dégradation des performances en mode commun et de la linéarité. Pour pallier cet inconvénient, on peut utiliser des structures à sorties symétriques, il convient alors d'ajouter un asservissement de courant de repos.

La figure 30 représente l'amplificateur à trans-

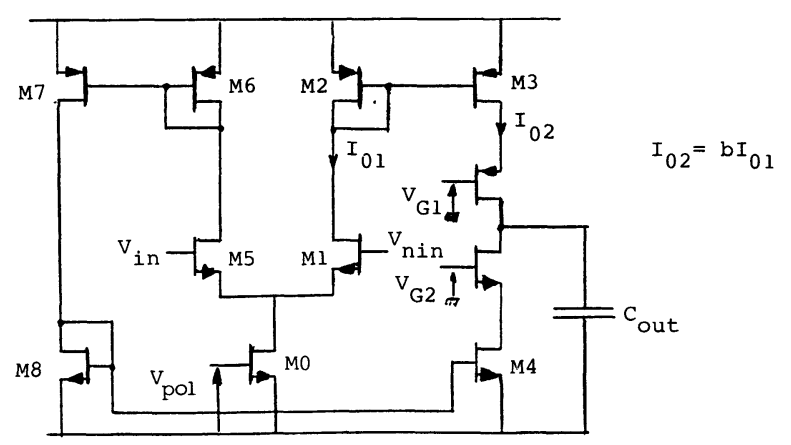

Fig. 29.

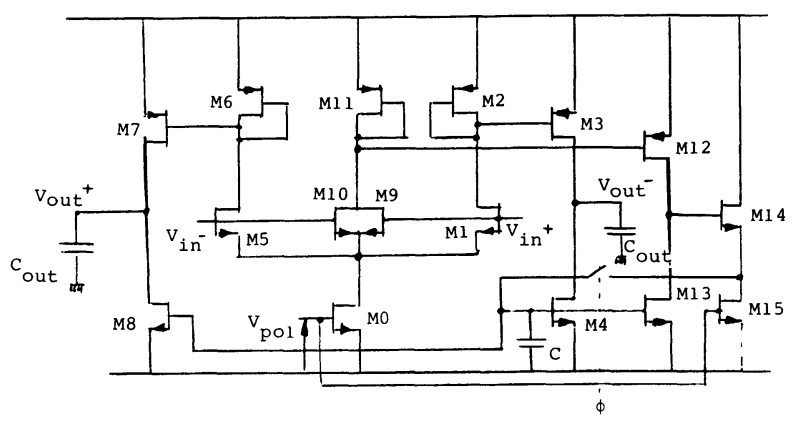

Fig. 30 . conductance symétrisé muni d'un asservissement de courant de repos échantillonné $\left(M_{9} \ldots M_{15}\right)$.

6.3.3.2 Amplificateur à courant de polarisation variable (Fig. 31). - L'augmentation des performances dynamiques transitoires à géométrie donnée d'une cellule amplificatrice passe par l'accroissement du courant de polarisation; l'augmentation du gain statique par sa diminution.

La satisfaction de cette double contrainte implique la variation du courant de polarisation au cours du temps.

Ainsi dans une application utilisant la commutation de capacités, à chaque coup d'horloge le courant de polarisation des amplificateurs doit subir un rapide accroissement pour réduire la durée du régime transitoire, puis, peu à peu être ramené à sa valeur de repos pour satisfaire la contrainte sur le gain statique.

Deux solutions pratiques ont été envisagées. La première [3] consiste à moduler le courant de polarisation par des pointes recurrentes synchronisées par l'horloge. La seconde consiste à asservir le courant de repos à l'aide de sources de différences de courant [7].

La première est potentiellement un peu plus rapide que la seconde puisque le contrôle du courant de polarisation s'effectue en boucle ouverte, elle est donc applicable au fonctionnement en comparateur de l'amplificateur. Elle nécessite la présence d'un signal d'horloge et. n'est pas optimisée en puissance consommée. La seconde implique que l'amplificateur soit bouclé.

La difficulté dans l'usage de telles structures réside dans l'absence de méthode simple d'optimisation de la cellule pour un cahier des charges donné

La figure 31 représente un amplificateur à transconductance asservi en courant de polarisation (a), une source de différences de courant (b) et un amplificateur dynamique (c).

\subsection{COMPARAISON ENTRE LES DIFFÉRENTES STRUCTURES.}

- Dans cette section, nous allons présenter un tableau récapitulatif montrant les valeurs des principales grandeurs caractéristiques des montages que nous avons décrits précédemment.

Les valeurs présentées sont les résultats de simulations effectuées à l'aide du programme SPICE 2 .

La comparaison porte sur les points suivants :

- le gain statique $A_{0}$

- la capacité de charge $C_{\text {out }}$

- la capacité de compensation $C_{\mathrm{c}}$

- la fréquence de transition $f_{\mathrm{T}}$

- la fréquence de coupure haute $F_{\mathrm{p}_{2}}$

- la marge de phase $\varphi_{\mathrm{m}}$

- le slew-rate $S R$

- la consommation. 

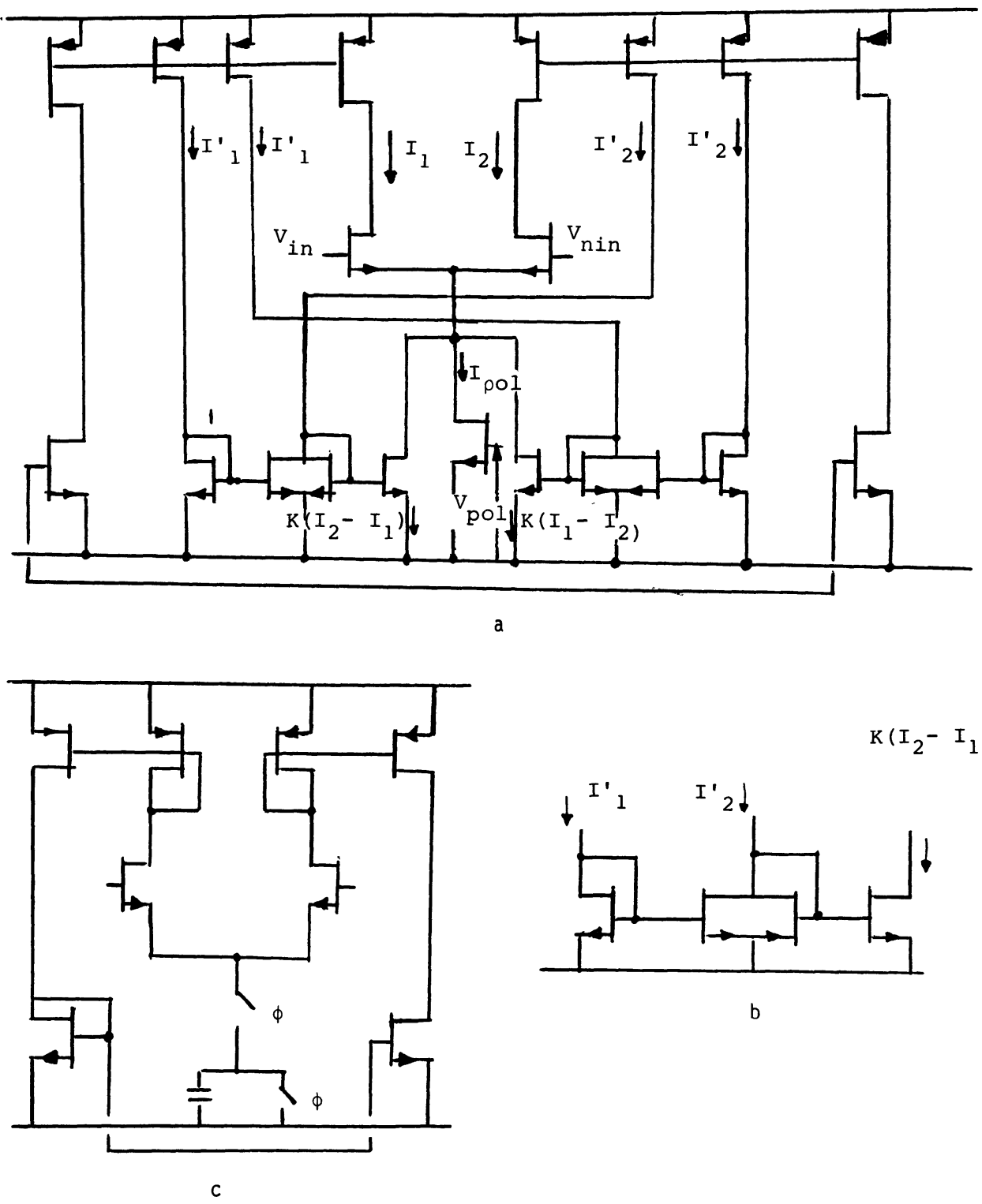

$K\left(I_{2}-I_{1}\right)$

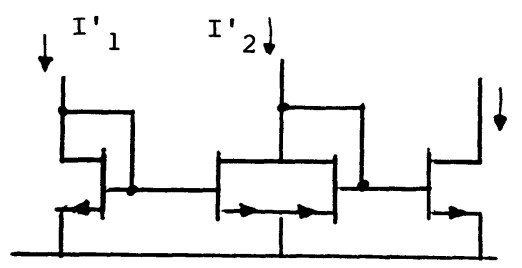

Fig. 31 .

\begin{tabular}{|c|c|c|c|c|c|c|c|c|c|}
\hline $\begin{array}{l}\text { Type } \\
\text { ampli. }\end{array}$ & $A_{0}$ & $C_{\text {out }} \mathrm{pF}$ & $C_{\mathrm{c}} \mathrm{pF}$ & $f_{\mathrm{T}} \mathrm{Hz}$ & $f_{\mathbf{p}_{2}} \mathrm{~Hz}$ & $\varphi_{\mathrm{m}}$ & $\begin{array}{c}S R \\
\mathrm{~V} / \mu \mathrm{s}\end{array}$ & $\begin{array}{c}\text { Consommation } \\
\mathrm{mW}\end{array}$ & \\
\hline $\begin{array}{l}\text { Traditionnel } \\
\text { Traditional }\end{array}$ & 33500 & 20 & 50 & $126 \mathrm{kHz}$ & $1,8 \mathrm{MHz}$ & $70^{\circ}$ & 0,4 & 0,4 & \multirow{2}{*}{ 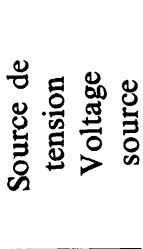 } \\
\hline $\begin{array}{l}\text { Traditionnel + cas- } \\
\text { code } \\
\text { Improved + cascode }\end{array}$ & 83000 & 20 & 5 & 1,2 MHz & $3 \mathrm{MHz}$ & $60^{\circ}$ & 2 & 0,9 & \\
\hline O.T.A. & 240 & 20 & - & $500 \mathrm{kHz}$ & $5 \mathrm{MHz}$ & $85^{\circ}$ & 1,6 & 0,65 & \multirow[b]{2}{*}{ 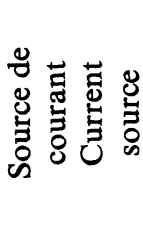 } \\
\hline $\begin{array}{l}\text { Cascode complémen- } \\
\text { taire } \\
\text { Complementary cas- } \\
\text { code }\end{array}$ & 250 & 20 & - & $500 \mathrm{kHz}$ & $10 \mathrm{MHz}$ & $88^{\circ}$ & 1,4 & 0,9 & \\
\hline
\end{tabular}




\section{Conclusion de l'étude comparative des structures de base.}

Dans l'amplificateur source de courant le second pôle dominant de la fonction de transfert $\left(\omega_{\mathrm{p}_{2}}\right)$ est fixé par la faible valeur des capacités parasites caractéristiques de la filière technologique.

$\mathrm{Au}$ contraire dans l'amplificateur source de tension c'est la capacité de charge, toujours élevée par rapport aux capacités parasites, qui en détermine la valeur.

C'est pourquoi les amplificateurs source de courant possèdent une potentialité transitoire intrinsèque en régime d'établissement supérieure à celle des amplificateurs source de tension.

- Par ailleurs à vitesse de balayage comparable, sur charge capacitive " utile " identique, les amplificateurs source de courant consomment moins d'énergie que les amplificateurs source de tension.

- D'autre part, les amplificateurs sources de courant peuvent être aisément utilisés en tant que cellules précaractérisées puisque leur comportement transitoire lors d'un transfert de charge peut être rendu indépendant de la capacité d'intégration $C_{\text {out }}$.

- Enfin, l'usage de l'asservissement du courant de polarisation permet dans l'amplificateur source de courant, de réaliser un compromis optimum temps de réponse consommation. Ces potentialités remarquables doivent toutefois être exploitées avec précaution car :

- L'amplificateur source de courant devient instable lorsque simultanément $C_{\text {in }}$ et $C_{\mathrm{s}}$ sont très faibles, $\omega_{\mathrm{T}}$ étant alors supérieur à $\omega_{\mathrm{p}_{2}}$. Pour éviter que de telles situations apparaissent lors des commutations des intercepteurs, il faut surcharger de manière permanente la sortie de l'amplificateur.

- La forte impédance de sortie des amplificateurs source de courant n'interdit pas les effets directs de l'entrée sur la sortie à travers le pont diviseur $C_{\text {out }}$, $C_{\mathrm{S}}$ qui se traduisent par des pointes de commutation (Fig. 20). Inexistant dans les amplificateurs source de tension, ce comportement nuisible peut être de nouveau réduit par la surcharge de la sortie de l'amplificateur source de courant.

- Enfin les amplificateurs " source de courant" conduisent à des systèmes affectés d'un bruit de commutation, d'une sensibilité aux variations des tensions d'alimentation supérieurs à ceux obtenus dans les systèmes fonctionnellement identiques utilisant les amplificateurs source de tension.

Cet inconvénient peut être largement réduit par l'utilisation de structures différentielles.

Le bon usage des amplificateurs source de courant nécessite donc l'emploi d'un simulateur électrique rendant bien compte du transfert de charge (ce que ne permettent pas les versions standards de SPICE II) et peut-être même l'emploi de maquettes sur table en appliquant une mise à l'échelle des capacités et des fréquences d'horloge.

\section{Annexe.}

Le transistor MOS en petits signaux.

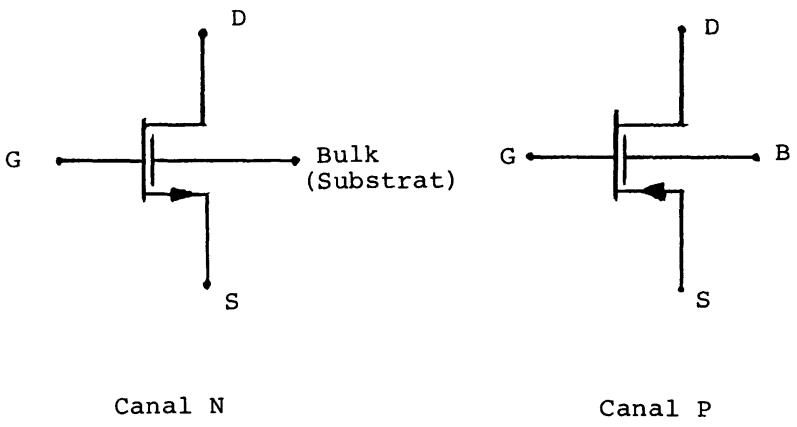

Fig. 32 .

Le fonctionnement du transistor MOS dans la région saturée, qui nous intéresse en analogique est modélisé par l'équation (1) :

$$
I_{\mathrm{DS}}=\mu C_{\mathrm{ox}} \frac{W}{2 L}\left(V_{\mathrm{GS}}-V_{\mathrm{T}}\right)^{2}\left(1+\lambda V_{\mathrm{DS}}\right)
$$

$\mu \quad$ : mobilité des porteurs dans le canal

$C_{\text {ox }} \quad$ : capacité d'oxyde de grille par unité de surface

$V_{\mathrm{T}} \quad$ : tension de seuil

$W, L$ : largeur, longueur électriques du canal

$\lambda \quad$ : coefficient de modulation de longueur $\mathrm{du}$ canal.

En linéarisant l'équation (1), on obtient le schéma équivalent petit signal.

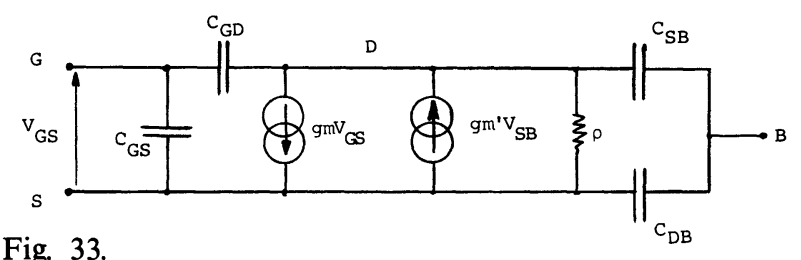

$g_{m}=\sqrt{2 \beta I_{0}}$ transconductance du transistor MOS

$\beta=\mu C_{\text {ox }} \frac{W}{L}$

$I_{0} \quad=$ courant de polarisation

$g_{m^{\prime}}=g_{m} \frac{\gamma}{2 \sqrt{V_{\mathrm{SB}}+2 \phi_{\mathrm{FP}}}}$ transconductance due à l'effet de substrat

$\gamma=$ coefficient d'effet de substrat

$\gamma=\frac{\sqrt{2 q N_{\mathrm{A}} \varepsilon_{0} \varepsilon_{\mathrm{SC}}}}{C_{\mathrm{ox}}}$

$N_{\mathrm{A}}=$ dopage du substrat

$\varepsilon_{\mathrm{SC}}=$ constante diélectrique du substrat

$q \quad=$ charge de l'électron

$C_{\mathrm{GS}}=\frac{2}{3} C_{\mathrm{ox}} W \cdot L+C$ recouvrement grille source

$C_{\mathrm{GD}}=C$ recouvrement grille drain

$C_{\mathrm{DB}}$ et $C_{\mathrm{SB}}=$ sont les capacités des jonctions drain substrat et source substrat

$\rho=\frac{1}{\lambda I_{0}}=$ résistance de sortie du MOS. 


\section{Bibliographie}

[1] Baillieu, F., Concina, S., Dupré, F., Les amplificateurs opérationnels NMOS, Onde Electrique (1983).

[2] Gray, R., Basic MOS.O.A. design : an overview, IEEE Press Analog MOS integrated circuits.

[3] Hosticka, Dynamic CMOS amplifiers, IEEE J. Solid State Circuits (1980).

[4] BlaCK, Allstot, ReED, High performance low power CMOS channel filter, IEEE J. Solid State Circuits (1980).

[5] Jolly, McCharles, $A$ low noise amplifier for switched capacitor filters, IEEE J. Solid State Circuits (1982).

[6] ChuAng, Analysis of the settling behavior of an O.A. IEEE J. Solid State Circuits (1982).

[7] Degrauwe, Rijmenants, Vittoz, Adaptative basing CMOS amplifiers, IEEE J. Solid State Circuits (1982).

[8] Aнyja, B. Y., An improved frequency compensation technique for CMOS operational amplifiers, IEEE J. Solid State Circuits (1983). 